\title{
An Adaptive Finite Element Method for Fredholm Integral Equations of the First Kind and its Verification on Experimental Data
}

NIKOLAY KOSHEV

LARISA BEILINA

Department of Mathematical Sciences Division of Mathematics

CHALMERS UNIVERSITY OF TECHNOLOGY UNIVERSITY OF GOTHENBURG

Gothenburg Sweden 2012 

Preprint 2012:20

\section{An Adaptive Finite Element Method for Fredholm Integral Equations of the First Kind and its Verification on Experimental Data}

Nikolay Koshev and Larisa Beilina

Department of Mathematical Sciences

Division of Mathematics

Chalmers University of Technology and University of Gothenburg

SE-412 96 Gothenburg, Sweden

Gothenburg, October 2012 
Preprint 2012:20

ISSN 1652-9715

Matematiska vetenskaper

Göteborg 2012 


\title{
AN ADAPTIVE FINITE ELEMENT METHOD FOR FREDHOLM INTEGRAL EQUATIONS OF THE FIRST KIND AND ITS VERIFICATION ON EXPERIMENTAL DATA
}

\author{
NIKOLAY KOSHEV* AND LARISA BEILINA **
}

\begin{abstract}
We propose an adaptive finite element method for the solution of a linear Fredholm integral equation of the first kind. We derive a posteriori error estimates in the functional to be minimized and in the regularized solution of this functional, and formulate corresponding adaptive algorithm. To do it we specify nonlinear results obtained in $[6,7,8,9,18]$ for the case of the linear bounded operator. Numerical experiments justify the efficiency of our a posteriori estimates applied both to the computationally simulated and experimental backscattered data measured in microtomography.
\end{abstract}

1. Introduction. In this work we consider a problem of the solution of a Fredholm integral equation of the first kind and propose an adaptive finite element method to solve it. Such problems arise in many applications of astrophysics [13], astronomy [3], image processing of smeared and defocused photography [19] and image processing in microtomography [20], spectroscopy in the backscattered electron signal [10], etc. An adaptive finite element method for a Fredholm integral equation of the second kind was considered in [1]. In this paper we solve a more complicated and different problem: we derive a posteriori error estimates for a Fredholm integral equation of the first kind which is an ill-posed problem. Thus, to solve this problem, we need minimize the corresponding Tikhonov functional.

We will consider a Fredholm integral equation of the first kind which takes the form

$$
\int_{D} K(t, s) z(s) d s=u(t), t \in D
$$

Here, $D$ is a closed bounded set in $\mathbb{R}^{n}, n=2,3$. It is assumed that the kernel $K(t, s)$ is the absolutely integrable function. Equation (1.1) can be written in an operator form as

$$
A(z)=u
$$

where $A: X \rightarrow Y$ and $X$ and $Y$ are complete metric spaces.

In the case when the kernel $K(t, s)$ in (1.1) is a smooth function then equations of type (1.1) are classified as ill-posed problems since the solution $z$ is sensitive to the small perturbations in the data function $u$, see $[15,22,13,25,26]$. If the kernel $K(t, s)$ is a singular function then the ill-posedness in (1.1) is feasible. One of the examples of this type equation is the boundary integral equations used for solution of elliptic partial differential equations, see [2] and references therein. In the current paper we consider the case when the operator $A^{-1}$ in (1.2) is not compact operator and thus we are dealing with a classic case of ill-posed problem [26].

Main novelty of our work is in the derivation of a posteriori error estimates for the Tikhonov functional and for the regularized solution of this functional. These estimates are derived not only on the conforming finite element meshes where continuous piecewise linear functions CG(1) are applied but even on the meshes with hanging nodes. Thus, we apply discontinuous finite element method DG(1) on these meshes. To do that we specify results of $[6,7,8,9,18]$ for the case of the linear Fredholm integral equation of the first kind. Because of the linearity, results of this paper sound more clear than those of previous works and proofs here are different from ones of $[6,7,8,9,18]$. Another novelty of this paper is that the Tikhonov regularization term is given in the $H^{1}$ norm, which is stronger than the $L_{2}$ norm being used in $[6,7,8,9,18]$. All these new moments causes additional difficulties, compared with $[6,7,8,9,18]$.

\footnotetext{
*Department of Physics, Penza State University of Architecture and Building, Russian Federation, email: nikolay.koshev@gmail.com

** Corresponding author, Department of Mathematical Sciences, Chalmers University of Technology and Gothenburg University, SE-42196 Gothenburg, Sweden, email: larisa@chalmers.se
} 
We note that in $[6,9]$ a posteriori error estimates were obtained for the regularized coefficient rather for the exact. In the follow-up work [18] the effort of the accuracy improvement of the regularized coefficient rather than the exact coefficient was explained. More precisely, from [18] follows that the regularized coefficient is closer to the exact coefficient than the first guess in the nonlinear case. Therefore, an improvement of the accuracy of the reconstruction of the regularized coefficient on the adaptively refined meshes leads to an improvement of the accuracy of the reconstruction of the exact solution. Similarly with $[6,9]$ we obtain new a posteriori error estimates for the regularized function.

The main concept of the adaptivity technique, which we apply to a Fredholm integral equation of the first kind, is following. In the case of ill-posed problems it is inefficient to use an exceedingly fine mesh in computations. The main idea of the adaptive finite element method is to obtain a good accuracy of solutions via local mesh refinements. In order to do it, we minimize the Tikhonov functional on a sequence of locally refined meshes. A posteriori error analysis developed in this paper addresses the main question in the adaptivity: where to refine the mesh locally in order to improve the resulting solution.

In our numerical examples we demonstrate the efficiency of the adaptivity technique for the image restoration problem of electron microscopy $[19,20]$. The goal of our tests is to restore blurred images obtained in electron microscopy and identify possible defects on them. To do that we apply adaptive algorithm of Section 9. Since in computational examples of Section 10 we work only with a finite dimensional space of standard piecewise linear finite elements, then we consider our problem in a finite dimensional space. However, the corresponding Fredholm integral equation of the first kind certainly inherits the ill-posed nature of its infinitely dimensional analog. Therefore, it is worth to consider the Tikhonov functional.

In Tests 1,2 of Section 10 we have applied computationally simulated data, while in Test 3 was used real measured data obtained by the microtomograph developed by Professor Eduard Rau at Moscow Lomonosov State University [19, 20]. Conclusion from these tests is that the local adaptive mesh refinement algorithm can significantly improve contrast of the blurred images. In Test 2 of Section 10 we compare the performance of our adaptive algorithm with performance of methods of $[20,21]$ on the reconstruction of the deconvolution function. Our computational tests show that an adaptive finite element method gives better stability in the reconstruction of the deconvolution function.

Comparison with other techniques which are used for solution of such kind of problems is presented in Section 10.4. We compare three different reconstruction methods: an adaptive finite element method of this paper, uniform grid deconvolution method of [19], and Bounded Total Variation method of [20]. Test 4 demonstrates that an adaptive finite element method gives better results in the reconstruction of the highintensive smeared images than other two reconstruction methods. However, the Bounded Total Variation Method of [20] performs better in the case of large noise level in the computational data on the reconstruction of non-intensive smeared images. Our conclusion is that the choice of the particular reconstruction method depends on the level of the noise in computational data and level of the smearing intensity of the image to be reconstructed.

We do not consider the case of the multi-connected computational domains in this work. However, based on the results of Test 4 in Section 10.4, it can be interesting task to consider such domains in a future research with different reconstruction methods applied in each domain.

An outline of this paper is as follows: In Section 2 we present the statement of the problem, the corresponding Tikhonov functional and it's Frechét derivative. In Section 3 we describe CG(1) and DG(1) finite element spaces and in Section 4 we present CG(1) and DG(1) finite element method for the Tikhonov functional. In Section 5 we demonstrate general framework for a posteriori error estimates. A posteriori error estimate for the regularized solution on a locally refined meshes is derived in Section 6 and a posteriori error estimate for the error in the Tikhonov functional is presented in Section 7. We formulate mesh refinement recommendations and adaptive algorithms in Sections 8, 9, correspondingly. Finally, in our concluding Section 10 we demonstrate results of the reconstruction in two dimensions using adaptive algorithms of Section 9 .

2. Notations used in the paper. We introduce first common notations which we are using in this paper. Let $\Omega \subset \mathbb{R}^{n}, n=2,3$ be a bounded domain with the piecewise-smooth boundary $\partial \Omega$. In our numerical 
experiments we work with piecewise smooth boundaries and this is one of discrepancies between the theory and its numerical implementation.

Let function $u(x), x=\left(x_{1}, \ldots, x_{n}\right) \in \Omega$ be a $k$ times continuously differentiable in $\Omega$. We denote the partial derivative of the order $|\alpha| \leq k$, of function $u$ by

$$
D^{\alpha} u=\frac{\partial^{|\alpha|} u}{\partial^{\alpha_{1}} x_{1} \ldots \partial^{\alpha_{n}} x_{n}},|\alpha|=\alpha_{1}+\ldots+\alpha_{n},
$$

where $\alpha=\left(\alpha_{1}, \ldots, \alpha_{n}\right)$ is such that $\alpha_{i} \geq 0$.

Denote $C^{k}(\bar{\Omega})$ the Banach space of functions $u(x)$ which are continuous in the closure $\Omega$ of the domain $\Omega$ together with their derivatives $D^{\alpha} u,|\alpha| \leq m$. The norm in this space is defined as

$$
\|u\|_{C^{k}(\Omega)}=\sum_{|\alpha| \leq m} \sup _{x \in \Omega}\left|D^{\alpha} u(x)\right|<\infty .
$$

Consider the Sobolev space $H^{k}(\Omega)$ of all functions with the norm defined as

$$
\|u\|_{H^{k}(\Omega)}^{2}=\sum_{|\alpha| \leq k} \int_{\Omega}\left|D^{\alpha} u\right|^{2} d x<\infty,
$$

where $D^{\alpha} u$ are weak derivatives of the function $u$.

$H^{k}(\Omega)$ is a Hilbert space with the inner product defined as

$$
(u, v)_{H^{k}(\Omega)}=\sum_{|\alpha| \leq k} \int_{\Omega} D^{\alpha} u D^{\alpha} v d x .
$$

In $L_{2}(\Omega)$ the inner product and the norm are defined as

$$
\begin{aligned}
(u, v) & =\int_{\Omega} u v d x, x \in \Omega, \\
\|u\|^{2} & =(u, u) .
\end{aligned}
$$

2.1. Statement of the problem. Let $H$ be the Hilbert space of functions defined in $\Omega$. Recall that we consider $\Omega \subset \mathbb{R}^{n}, n=2,3$ which is a bounded domain with the piecewise-smooth boundary $\partial \Omega$. Our goal is to solve a Fredholm integral equation of the first kind

$$
\int_{\Omega} \rho(x, y) z(x) d x=u(y) \quad y \in \Omega
$$

where $u(y) \in L_{2}(\Omega), z(x) \in H, \rho(x, y) \in C^{k}(\Omega \times \Omega), k \geq 0$ is the kernel of the integral equation.

We can rewrite $(2.1)$ in an operator form as

$$
A(z)=u
$$

with an operator $A: H \rightarrow L_{2}(\Omega)$ defined as

$$
A(z):=\int_{\Omega} \rho(x, y) z(x) d x .
$$

The Problem (P).

Let the function $z(x) \in H$ in the equation

$$
\int_{\Omega} \rho(x, y) z(x) d x=u(y) \quad y \in \Omega
$$

be unknown in the domain $\Omega$. Determine the function $z(x) \in H$ for $x \in \Omega$ assuming that functions $\rho(x, y) \in$ $C^{k}(\Omega \times \Omega), k \geq 0$ and $u(y) \in L_{2}(\Omega)$ in (2.4) are known.

Although the function $A(z) \in C^{k}(\Omega), k \geq 0$ we assume in (2.3) that $u \in L_{2}(\Omega)$. The reason of this is that the right hand side of this equation can be noisy. 
2.2. The Tikhonov functional. We assume that the right hand side of (2.4) is given with the small parameter $\delta \in(0,1)$ which characterizes the level of the error in data. Let $u^{*}$ be the perfect noiseless right hand side of (2.4) which corresponds to the exact solution $z^{*}$ of $(2.2)$ such that

$$
A\left(z^{*}\right)=u^{*}, \quad\left\|u-u^{*}\right\|_{L_{2}(\Omega)} \leq \delta .
$$

Introduce the operator $F: H \rightarrow L_{2}$ such that

$$
F(z):=A z-u \text {. }
$$

Hence $A z^{*}-u^{*}=0$. Since $\left\|u-u^{*}\right\|_{L_{2}(\Omega)} \leq \delta$ then

$$
\left\|F\left(z^{*}\right)\right\|_{L_{2}(\Omega)} \leq \delta .
$$

Recall that the problem of solution of the operator equation (2.2) is a classical ill-posed problem [24] since the operator $A^{-1}$ may not be compact. Thus, we assume that there exists the exact solution $z^{*}$ to our problem $\mathbf{P}$ but we never will get this solution in computations. Because of that we call by the regularized solution $z_{\alpha}$ some approximation of the unknown exact solution $z^{*}$ which is satisfied to the requirements of closeness to the exact solution $z^{*}$ and stability with respect to the small errors of the right-hand side $u(y)$ of equation (2.2). Algorithm for solution of the equation (2.2) can be written in the form $z_{\alpha}=R(\delta, \alpha) u$, where $R(\delta, \alpha): L_{2} \rightarrow H$ is regularization operator, see in [24] for more information about construction of this operator. When we apply this operator to the solution of the ill-posed problem we get a regularization algorithm. In this paper we use Tikhonov regularization algorithm which is based on the minimization of the Tikhonov functional. Thus, to find regularized solution $z_{\alpha}$ of equation (2.4), we take $H=H^{1}$ and minimize the Tikhonov regularization functional $M_{\alpha}(z)$ in the form

$$
\begin{gathered}
M_{\alpha}(z)=\frac{1}{2}\|F(z)\|_{L_{2}(\Omega)}^{2}+\frac{\alpha}{2}\left\|z-z_{0}\right\|_{H^{1}(\Omega)}^{2}, \\
M_{\alpha}: H^{1} \rightarrow \mathbb{R}, \quad z_{0} \in H^{1},
\end{gathered}
$$

where $\alpha=\alpha(\delta)>0$ is a small regularization parameter. The choice of the point $z_{0}$ and the regularization parameter $\alpha$ depends on the concrete minimization problem and this is outside of the scope of this publication. Usually $z_{0}$ is a good first approximation for the exact solution $z^{*}$.

From [24] follows that an algorithm for solution of the equation (2.2) which is based on the minimization of the Tikhonov functional (2.8) is the regularization algorithm, and the element $z_{\alpha} \in H^{1}$ where the functional (2.8) reaches its minimum is the regularized solution.

Let us consider an important class of Fredholm integral equations of the first kind - the convolution equation. These equations can be presented in the form (2.2) with the convolution operator $A: H^{1} \rightarrow L_{2}(\Omega)$ defined by

$$
A(z):=\int_{\Omega} \rho(y-x) z(x) d x,
$$

where $\rho(y-x) \in C^{k}(\Omega \times \Omega), k \geq 0, \quad z(x) \in H^{1}$. Then using the convolution theorem and properties of the Fourier transform [25] we obtain the minimum $z(x) \in H^{1}$ of the functional (2.8) given by

$$
z(x)=F^{-1}\left(\frac{\hat{u}(\omega) \hat{\rho}^{*}(\omega)}{|\hat{\rho}(\omega)|^{2}+\alpha\left(1+\omega^{2}\right)}\right),
$$

where $\hat{f}(\omega)$ denotes the Fourier transform $F(f)(\omega)$ of the function $f(\omega)$ defined by

$$
\hat{f}(\omega):=F(f)(\omega)=\frac{1}{(2 \pi)^{n}} \int_{\mathbb{R}^{n}} f(x) e^{-i \omega x} d x .
$$

We consider the convolution equation (2.9) in our numerical examples of Section 10. We note that our a posteriori error estimates are valid also for this type of equation. 
2.3. The Fréchet Derivative and the convexity of the Tikhonov Functional. For the convenience of the presentation we introduce more general form of the Tikhonov functional (2.8). This is done for presentation of the general form of the convexity property of this functional. Let $W_{1}, W_{2}, Q$ be three Hilbert spaces, $Q \subseteq W_{1}$ as a set, the norm in $Q$ is stronger than the norm in $W_{1}$ and $\bar{Q}=W_{1}$, where the closure is understood in the norm of $W_{1}$. We denote scalar products and norms in these spaces as

$$
\begin{gathered}
(\cdot, \cdot),\|\cdot\| \text { for } W_{1} \\
(\cdot, \cdot)_{2},\|\cdot\|_{2} \text { for } W_{2} \\
\text { and }[\cdot, \cdot],[\cdot] \text { for } Q .
\end{gathered}
$$

Let $A: W_{1} \rightarrow W_{2}$ be a bounded linear operator. Our goal is to find the function $z \in Q$ which minimizes the Tikhonov functional

$$
\begin{gathered}
J_{\alpha}(z): Q \rightarrow \mathbb{R}, \\
J_{\alpha}(z)=\frac{1}{2}\|A z-u\|_{2}^{2}+\frac{\alpha}{2}\left[z-z_{0}\right]^{2}, u \in W_{2} ; z, z_{0} \in Q,
\end{gathered}
$$

where $\alpha \in(0,1)$ is the regularization parameter.

To do that we search for a stationary point of the above functional with respect to $z$ satisfying $\forall b \in Q$

$$
J_{\alpha}^{\prime}(z)(b)=0 .
$$

where $J_{\alpha}^{\prime}(z)$ is the Fréchet derivative of the functional (2.13) and $J_{\alpha}^{\prime}(z)(b)$ means that $J_{\alpha}^{\prime}$ acts on $b$.

The following lemma is well known [4] and we present it for the particular case when the operator $A: L_{2} \rightarrow L_{2}$. In the case when $A: H^{1} \rightarrow L_{2}$ the explicit derivation of the Fréchet derivative of the functional (2.13) is technically more complicated problem because of the presence of $H^{1}$ norm in the regularization term of the functional (2.13), and we omit here explicit presentation of the Fréchet derivative of the functional (2.13) in this case. However, in our proofs of Theorems 1 and 2 we mean that the Fréchet derivative of the functional (2.12) is given in $H^{1}$ norm when the operator $A: H^{1} \rightarrow L_{2}$.

Lemma 1. Let $A: L_{2} \rightarrow L_{2}$ be a bounded linear operator. Then the Fréchet derivative of the functional (2.12) is

$$
J_{\alpha}^{\prime}(z)(b)=\left(A^{*} A z-A^{*} u, b\right)+\alpha\left[z-z_{0}, b\right], \forall b \in Q .
$$

In particular, for the integral operator (2.4) we have

$$
\begin{gathered}
J_{\alpha}^{\prime}(z)(b)=\int_{\Omega} b(s)\left[\int_{\Omega} z(y)\left(\int_{\Omega} \rho(x, y) \rho(x, s) d x\right) d y-\int_{\Omega} \rho(x, s) u(x) d x\right] d s \\
+\alpha\left[z-z_{0}, b\right], \forall b \in Q .
\end{gathered}
$$

Lemma 2 is also well known since $A: W_{1} \rightarrow W_{2}$ is a bounded linear operator. We again formulate this lemma only for our specific case, referring to [25] for a more general case. The situation is naturally more complicated for a nonlinear operator, and we refer to [6] for this case.

Lemma 2. Let the operator $A: W_{1} \rightarrow W_{2}$ be a bounded linear operator which has the Fréchet derivative of the functional (2.12). Then the functional $J_{\alpha}(z)$ is strongly convex on the space $Q$ and

$$
\left(J_{\alpha}^{\prime}(x)-J_{\alpha}^{\prime}(z), x-z\right) \geq \alpha[x-z]^{2}, \forall x, z \in Q .
$$


It is known from the theory of convex optimization that Lemma 2 implies existence and uniqueness of the global minimizer $z_{\alpha} \in Q$ of the functional $J_{\alpha}$ defined in (2.12) such that

$$
J_{\alpha}\left(z_{\alpha}\right)=\inf _{z \in Q} J_{\alpha}(z) .
$$

It is well known that the operator $F$ is Lipschitz continuous

$$
\left\|F\left(z_{1}\right)-F\left(z_{2}\right)\right\| \leq\|A\| \cdot\left\|z_{1}-z_{2}\right\| \quad \forall z_{1}, z_{2} \in H .
$$

We also introduce new constant $D=D(\|A\|, \alpha)=$ const. $>0[4,5]$ such that

$$
\left\|J_{\alpha}^{\prime}\left(z_{1}\right)-J_{\alpha}^{\prime}\left(z_{2}\right)\right\| \leq D\left\|z_{1}-z_{2}\right\|, \forall z_{1}, z_{2} \in H .
$$

3. The finite element spaces. Following [17] we discretize our bounded domain $\Omega \subset \mathbb{R}^{n}, n=2,3$ by an unstructured mesh $T$ using non-overlapping elements $K$. In $\mathbb{R}^{3}$ the elements $K$ are tetrahedrons and in $\mathbb{R}^{2}$ the elements $K$ are triangles such that $T=K_{1}, \ldots, K_{l}$, where $l$ is the total number of elements in $\Omega$, and

$$
\Omega=\cup_{K \in T} K=K_{1} \cup K_{2} \ldots \cup K_{l} .
$$

We associate with the triangulation $T$ the mesh function $h=h(x)$ which is a piecewise-constant function such that

$$
h(x)=h_{K} \quad \forall K \in T
$$

where $h_{K}$ is the diameter of $K$ which we define as the longest side of $K$.

Let $r^{\prime}$ be the radius of the maximal circle/sphere contained in the element $K$. We make the following shape regularity assumption for every element $K \in T$

$$
a_{1} \leq h_{K} \leq r^{\prime} a_{2} ; \quad a_{1}, a_{2}=\text { const. }>0 .
$$

We introduce now the finite element space $V_{h}$ as

$$
V_{h}=\left\{v(x) \in V: v \in C(\Omega),\left.v\right|_{K} \in P_{1}(K) \forall K \in T\right\},
$$

where $P_{1}(K)$ denotes the set of piecewise-linear functions on $K$ with

$$
V=\left\{v(x): v(x) \in H^{1}(\Omega)\right\} .
$$

The finite dimensional finite element space $V_{h}$ is constructed such that $V_{h} \subset V$. The finite element method which uses piecewise-linear test functions we call continuous Galerkin, or CG(1) method. CG(1) can be applied on the conforming meshes.

In a general case we also allow meshes in a space with hanging nodes and assume that the local mesh size has bounded variation in such meshes. This means that there exists a constant $\gamma>0$ such that $\gamma h_{K^{+}} \leq h_{K} \leq \gamma^{-1} h_{K^{+}}$for all neighboring elements $K^{-}$and $K^{+}$. Let $S$ be the internal face of the nonempty intersection of the boundaries of two neighboring elements $K^{+}$and $K^{-}$. We denote the jump of the function $v_{h}$ computed from the two neighboring elements $K^{+}$and $K^{-}$sharing the common side $S$ as

$$
\left[v_{h}\right]=v_{h}^{+}-v_{h}^{-} .
$$

We introduce the discontinuous finite element space $W_{h}$ on such meshes as

$$
W_{h}=\left\{v(x) \in V:\left.v\right|_{K} \in D P_{1}(K) \forall K \in T\right\},
$$

where $D P_{1}(K)$ denotes the set of discontinuous linear functions on $K$. The finite element space $W_{h}$ is constructed such that $W_{h} \subset V$. The finite element method which uses discontinuous piecewise-linear functions is called DG(1) method. 
Let $M$ be a subspace of the space $V$. Let $P_{h}: V \rightarrow M$ for $\forall M \subset V$, be the operator of the orthogonal projection of $V$ on $M$. Let the function $f \in H^{1}(\Omega) \cap C(\Omega)$ and $\partial_{x_{i}} f_{x_{i}} \in L_{\infty}(\Omega)$. We define by $f_{k}^{I}$ the standard interpolant [12] on triangles/tetrahedra of the function $f \in H$. Then by one of properties of the orthogonal projection

$$
\left\|f-P_{h} f\right\|_{L_{2}(\Omega)} \leq\left\|f-f_{k}^{I}\right\|_{L_{2}(\Omega)} .
$$

It follows from formula 76.3 of [12] that

$$
\left\|f-P_{h} f\right\|_{L_{2}(\Omega)} \leq C_{I}\|h \nabla f\|_{L_{2}(\Omega)}, \forall f \in V .
$$

where $C_{I}=C_{I}(\Omega)$ is positive constant depending only on the domain $\Omega$.

4. A finite element method. To formulate a CG(1) for equation (2.14) we recall the definition of the space $V_{h}$. The CG(1) finite element method then reads: find $z_{h} \in V_{h}$ such that

$$
J_{\alpha}^{\prime}\left(z_{h}\right)(b)=0 \quad \forall b \in V_{h} .
$$

Similarly, for DG(1) for equation (2.14) we recall the definition of the space $W_{h}$. The DG(1) finite element method then reads: find $z_{h} \in W_{h}$ such that

$$
J_{\alpha}^{\prime}\left(z_{h}\right)(b)=0 \quad \forall b \in W_{h} .
$$

5. General framework of a posteriori error estimate. Our goal is to present a posteriori error estimate for two kinds of error:

- For the error $\left|J_{\alpha}\left(z_{\alpha}\right)-J_{\alpha}\left(z_{h}\right)\right|$ in the Tikhonov functional (2.12).

- For the error $\left|z_{\alpha}-z_{h}\right|$ in the regularized solution of this functional $z_{\alpha}$.

To achieve the first goal, we note that

$$
J_{\alpha}\left(z_{\alpha}\right)-J_{\alpha}\left(z_{h}\right)=J_{\alpha}^{\prime}\left(z_{h}\right)\left(z_{\alpha}-z_{h}\right)+R\left(z_{\alpha}, z_{h}\right)
$$

where $R\left(z_{\alpha}, z_{h}\right)$ is the second order remainder term. We assume that $z_{h}$ is located in the small neighborhood of the regularized solution $z_{\alpha}$. Thus, the term $R\left(z_{\alpha}, z_{h}\right)$ is small and we can neglect it.

We now use the Galerkin orthogonality principle

$$
J_{\alpha}^{\prime}\left(z_{h}\right)(b)=0 \quad \forall b \in V_{h} \quad \text { or } \quad \forall b \in W_{h}
$$

together with the splitting

$$
z_{\alpha}-z_{h}=\left(z_{\alpha}-z_{\alpha}^{I}\right)+\left(z_{\alpha}^{I}-z_{h}\right)
$$

where $z_{\alpha}^{I} \in V_{h}$ or $z_{\alpha}^{I} \in W_{h}$ is the interpolant of $z_{\alpha}$, and get the following error representation:

$$
J_{\alpha}\left(z_{\alpha}\right)-J_{\alpha}\left(z_{h}\right) \approx J_{\alpha}^{\prime}\left(z_{h}\right)\left(z_{\alpha}-z_{\alpha}^{I}\right) .
$$

A posteriori error estimate (5.4) involves the derivative of the Tikhonov functional $J_{\alpha}^{\prime}\left(z_{h}\right)$ which represents residual multiplied by weights $z_{\alpha}-z_{\alpha}^{I}$.

To derive the error $z_{\alpha}-z_{h}$ in the regularized solution $z_{\alpha}$ we use the convexity property of the Tikhonov functional (2.12) together with the interpolation property (3.6).

We now make both error estimates more explicit for the case of CG(1) and DG(1) applied to solution of $(2.4)$. 
6. A posteriori error estimate of the regularized solution on refined meshes. In this section we formulate theorem for a posteriori error estimate for the regularized solution $z_{\alpha}$ for the case of the functional $M_{\alpha}$ defined in (2.8). This Theorem is also valid in a common case of the functional $J_{\alpha}(z)$ defined in (2.13). To reduce notations during the proof of this theorem we define the scalar product $(\cdot, \cdot)_{H^{1}}$ as $(\cdot, \cdot)$.

Theorem 1

Let $z_{h} \in W_{h}$ be a finite element approximation of the regularized solution $z_{\alpha} \in H^{2}(\Omega)$ on the finite element mesh $T$ with the mesh function $h$. Then there exists a constant $D$ defined by (2.17) such that the following a posteriori error estimate for the regularized solution $z_{\alpha}$ holds

$$
\left\|z_{h}-z_{\alpha}\right\|_{H^{1}(\Omega)} \leq \frac{D}{\alpha} C_{I}\left(\left\|h z_{h}\right\|_{L_{2}(\Omega)}+\left\|\left[z_{h}\right]\right\|_{L_{2}(\Omega)}+C \max _{S \subset \partial K} h_{K}^{-1}\left\|\left[\partial_{S} z_{h}\right]\right\|_{L_{2}(\Omega)}\right) \forall z_{h} \in W_{h} .
$$

In the case when $z_{h} \in V_{h}$ we have a posteriori error estimate

$$
\left\|z_{h}-z_{\alpha}\right\|_{H^{1}(\Omega)} \leq \frac{D}{\alpha} C_{I}\left\|h z_{h}\right\|_{L_{2}(\Omega)} .
$$

\section{Proof.}

Let $z_{h}$ be the minimizer of the Tikhonov functional (2.8). The existence and uniqueness of this minimizer is guaranteed by Lemma 2. Since by Lemma 2 the functional (2.8) is strongly convex on the space $H^{1}$ with the strong convexity constant $\alpha$, then this fact implies that

$$
\alpha\left\|z_{h}-z_{\alpha}\right\|_{H^{1}(\Omega)}^{2} \leq\left(M_{\alpha}^{\prime}\left(z_{h}\right)-M_{\alpha}^{\prime}\left(z_{\alpha}\right), z_{h}-z_{\alpha}\right) .
$$

where $M_{\alpha}^{\prime}\left(z_{h}\right), M_{\alpha}^{\prime}\left(z_{\alpha}\right)$ are the Fréchet derivatives of the functional (2.8) for the operator $A: H^{1} \rightarrow L_{2}$.

Since $z_{\alpha}$ is the minimizer on the set $H^{1}$, then

$$
\left(M_{\alpha}^{\prime}\left(z_{\alpha}\right), z\right)=0, \forall z \in H^{1} .
$$

Using (6.2) with the splitting

$$
z_{h}-z_{\alpha}=\left(z_{h}-P_{h} z_{\alpha}\right)+\left(P_{h} z_{\alpha}-z_{\alpha}\right),
$$

together with the Galerkin orthogonality principle

$$
\left(M_{\alpha}^{\prime}\left(z_{h}\right)-M_{\alpha}^{\prime}\left(z_{\alpha}\right), z_{h}-P_{h} z_{\alpha}\right)=0
$$

such that either $\left(z_{h}, P_{h} z_{\alpha}\right) \in V_{h}$ for CG(1) or $\left(z_{h}, P_{h} z_{\alpha}\right) \in W_{h}$ for DG(1), and we obtain

$$
\alpha\left\|z_{h}-z_{\alpha}\right\|_{H^{1}}^{2} \leq\left(M_{\alpha}^{\prime}\left(z_{h}\right)-M_{\alpha}^{\prime}\left(z_{\alpha}\right), P_{h} z_{\alpha}-z_{\alpha}\right) .
$$

We can estimate the right hand side of (6.4) using (2.17) as

$$
\left(M_{\alpha}^{\prime}\left(z_{h}\right)-M_{\alpha}^{\prime}\left(z_{\alpha}\right), P_{h} z_{\alpha}-z_{\alpha}\right) \leq D\left\|z_{h}-z_{\alpha}\right\|_{H^{1}(\Omega)}\left\|P_{h} z_{\alpha}-z_{\alpha}\right\|_{H^{1}(\Omega)} .
$$

Substituting above equation into (6.4) we obtain

$$
\left\|z_{h}-z_{\alpha}\right\|_{H^{1}(\Omega)} \leq \frac{D}{\alpha}\left\|P_{h} z_{\alpha}-z_{\alpha}\right\|_{H^{1}(\Omega)} .
$$

Using the interpolation property

$$
\left\|P_{h} z_{\alpha}-z_{\alpha}\right\|_{H^{1}(\Omega)} \leq C_{I}\left\|h z_{\alpha}\right\|_{H^{2}(\Omega)}
$$

we obtain a posteriori error estimate for the regularized solution with the interpolation constant $C_{I}$ :

$$
\left\|z_{h}-z_{\alpha}\right\|_{H^{1}(\Omega)} \leq \frac{D}{\alpha}\left\|P_{h} z_{\alpha}-z_{\alpha}\right\|_{H^{1}(\Omega)} \leq \frac{D}{\alpha} C_{I}\left\|h z_{\alpha}\right\|_{H^{2}(\Omega)} .
$$


We can estimate $\left\|h z_{\alpha}\right\|_{H^{2}(\Omega)}$ as

$$
\begin{aligned}
& \left\|h z_{\alpha}\right\|_{H^{2}(\Omega)} \leq \sum_{K}\left\|h_{K} z_{\alpha}\right\|_{H^{2}(K)}=\sum_{K}\left\|\left(z_{\alpha}+\nabla z_{\alpha}+D^{2} z_{\alpha}\right) h_{K}\right\|_{L_{2}(K)} \\
& \leq \sum_{K}\left(\left\|z_{h} h_{K}\right\|_{L_{2}(K)}+\left\|\frac{\left[z_{h}\right]}{h_{K}} h_{K}\right\|_{L_{2}(K)}+\left\|\frac{\left[\partial_{\left.n_{K} z_{h}\right]}\right.}{h_{K}} h_{K}\right\|_{L_{2}(\partial K)}\right) \\
& \leq\left\|h z_{h}\right\|_{L_{2}(\Omega)}+\sum_{K}\left(\left\|\left[z_{h}\right]\right\|_{L_{2}(K)}+\left\|\left[\partial_{n_{K}} z_{h}\right]\right\|_{L_{2}(\partial K)}\right) .
\end{aligned}
$$

We denote in (6.6) by $D^{2} z_{\alpha}$ the second order derivatives of $z_{\alpha}, \partial K$ denotes the boundary of the element $K$, $\left[\partial_{n_{K}} z_{h}\right]$ is the jump of the normal derivative of the function $z_{h}$ in the outward direction $n_{K},\left[z_{h}\right]$ is the jump of the function $z_{h}$ over the element $K, h_{K}$ is the diameter of the element $K$. In (6.6) we also used the facts that $[16]$

$$
\left|\nabla z_{\alpha}\right| \leq \frac{\left|\left[z_{h}\right]\right|}{h_{K}}
$$

and

$$
\left|D^{2} z_{\alpha}\right| \leq \frac{\left|\left[\partial_{n_{K}} z_{h}\right]\right|}{h_{K}}
$$

We now estimate the third and second terms in the last row of (6.6). We take sum over the element boundaries such that the every internal side $S$ of the element $K$ occurs twice. We denote by $\partial_{S} z_{h}$ the derivative of the function $z_{h}$ in one of the normal directions of every side $S$ to get

$$
\sum_{K}\left\|\left[\partial_{n_{K}} z_{h}\right]\right\|_{L_{2}(\partial K)}=\sum_{S}\left\|\left[\partial_{S} z_{h}\right]\right\|_{L_{2}(S)},
$$

where $\left[\partial_{S} z_{h}\right]$ is the jump in the derivative $\partial_{S} z_{h}$ computed from the two triangles sharing the same side $S$. We now distribute every jump equally over the two triangles sharing the side $S$ and return to the sum over the elements boundaries $\partial K$ to get

$$
\sum_{S}\left\|\left[\partial_{S} z_{h}\right]\right\|_{L_{2}(S)}=\sum_{K} \frac{1}{2} h_{K}^{-1}\left\|\left[\partial_{S} z_{h}\right] h_{K}\right\|_{L_{2}(\partial K)} \cdot
$$

Now we replace norms over the element boundaries $\partial K$ by norm over the domain $\Omega$ to get

$$
\left|\sum_{K} \frac{1}{2} h_{K}^{-1}\left\|\left[\partial_{S} z_{h}\right] h_{K}\right\|_{L_{2}(\partial K)}\right| \leq C \max _{S \subset \partial K} h_{K}^{-1}\left\|\left[\partial_{S} z_{h}\right]\right\|_{L_{2}(\Omega)}
$$

with the constant $C>0$.

Substituting the above estimates into the right hand side of (6.6) we get

$$
\left\|z_{h}-z_{\alpha}\right\|_{H^{1}(\Omega)} \leq \frac{D}{\alpha} C_{I}\left\|h z_{h}\right\|_{L_{2}(\Omega)}+\frac{D}{\alpha} C_{I}\left(\left\|\left[z_{h}\right]\right\|_{L_{2}(\Omega)}+C \max _{S \subset \partial K} h_{K}^{-1}\left\|\left[\partial_{S} z_{h}\right]\right\|_{L_{2}(\Omega)}\right) \forall z_{h} \in W_{h} .
$$

In the case when $z_{h} \in V_{h}$ terms with jumps disappear and we have a posteriori error estimate

$$
\left\|z_{h}-z_{\alpha}\right\|_{H^{1}(\Omega)} \leq \frac{D}{\alpha} C_{I}\left\|h z_{h}\right\|_{L_{2}(\Omega)}
$$


7. A posteriori error estimates for the functional (2.8). In Theorem 2 we derive a posteriori error estimates for the error in the Tikhonov functional (2.8) on the finite element mesh $T$. This Theorem is also valid in a common case of the functional $J_{\alpha}(z)$ in (2.13).

\section{Theorem 2}

Suppose that there exists minimizer $z_{\alpha} \in H^{2}(\Omega)$ of the functional $M_{\alpha}$ on the set $V$ and mesh $T$. Suppose also that there exists finite element approximation $z_{h}$ of a minimizer $z_{\alpha}$ of $M_{\alpha}$ on the set $W_{h}$ and mesh $T$ with the mesh function $h$. Then the following approximate a posteriori error estimate for the error $e=\left|M_{\alpha}\left(z_{\alpha}\right)-M_{\alpha}\left(z_{h}\right)\right|$ in the Tikhonov functional (2.8) holds

$$
e=\left|M_{\alpha}\left(z_{\alpha}\right)-M_{\alpha}\left(z_{h}\right)\right| \leq C_{I}\left\|M_{\alpha}^{\prime}\left(z_{h}\right)\right\|_{H^{1}(\Omega)}\left(\left\|h z_{h}\right\|_{L_{2}(\Omega)}+\left\|\left[z_{h}\right]\right\|_{L_{2}(\Omega)}+C \max _{S \subset \partial K} h_{K}^{-1}\left\|\left[\partial_{S} z_{h}\right]\right\|_{L_{2}(\Omega)}\right) .
$$

In the case when the finite element approximation $z_{h} \in V_{h}$ we have following a posteriori error estimate

$$
e=\left|M_{\alpha}\left(z_{\alpha}\right)-M_{\alpha}\left(z_{h}\right)\right| \leq C_{I}\left\|M_{\alpha}^{\prime}\left(z_{h}\right)\right\|_{H^{1}(\Omega)}|| h z_{h} \|_{L_{2}(\Omega)} .
$$

\section{Proof}

By definition of the Frechét derivative we can write that on the mesh $T$ we have

$$
M_{\alpha}\left(z_{\alpha}\right)-M_{\alpha}\left(z_{h}\right)=M_{\alpha}^{\prime}\left(z_{h}\right)\left(z_{\alpha}-z_{h}\right)+R\left(z_{\alpha}, z_{h}\right)
$$

where by Lemma $1 R\left(z_{\alpha}, z_{h}\right)=O\left(\left(z_{\alpha}-z_{h}\right)^{2}\right), \quad\left(z_{\alpha}-z_{h}\right) \rightarrow 0 \quad \forall z_{\alpha}, z_{h} \in V$ and $M_{\alpha}^{\prime}\left(z_{h}\right)$ is the Fréchet derivative of the functional (2.8) for the operator $A: H^{1} \rightarrow L_{2}$. The term $R\left(z_{\alpha}, z_{h}\right)$ is small since we assume that $z_{h}$ is the minimizer of the Tikhonov functional on the mesh $T$ and this minimizer is located in a small neighborhood of the regularized solution $z_{\alpha}$. Thus, we can neglect $R$ in (7.3), see similar results for the case of a general nonlinear operator equation in $[4,6]$. Next, we use the splitting

$$
z_{\alpha}-z_{h}=z_{\alpha}-z_{\alpha}^{I}+z_{\alpha}^{I}-z_{h}
$$

and the Galerkin orthogonality [12]

$$
M_{\alpha}^{\prime}\left(z_{h}\right)\left(z_{\alpha}^{I}-z_{h}\right)=0 \forall z_{\alpha}^{I}, z_{h} \in W_{h}
$$

to get

$$
M_{\alpha}\left(z_{\alpha}\right)-M_{\alpha}\left(z_{h}\right) \leq M_{\alpha}^{\prime}\left(z_{h}\right)\left(z_{\alpha}-z_{\alpha}^{I}\right)
$$

where $z_{\alpha}^{I}$ is a standard interpolant of $z_{\alpha}$ on the mesh $T$ [12]. We have that

$$
\left|M_{\alpha}\left(z_{\alpha}\right)-M_{\alpha}\left(z_{h}\right)\right| \leq\left\|M_{\alpha}^{\prime}\left(z_{h}\right)\right\|_{H^{1}(\Omega)}|| z_{\alpha}-z_{\alpha}^{I} \|_{H^{1}(\Omega)},
$$

where the term $\left\|z_{\alpha}-z_{\alpha}^{I}\right\|_{H^{1}(\Omega)}$ in the right hand side of the above inequality can be estimated through the interpolation estimate with the constant $C_{I}$

$$
\left\|z_{\alpha}-z_{\alpha}^{I}\right\|_{H^{1}(\Omega)} \leq C_{I}\left\|h z_{\alpha}\right\|_{H^{2}(\Omega)} .
$$

Substituting above estimate into (7.7) we get

$$
\left|M_{\alpha}\left(z_{\alpha}\right)-M_{\alpha}\left(z_{h}\right)\right| \leq C_{I}\left\|M_{\alpha}^{\prime}\left(z_{h}\right)\right\|_{H^{1}(\Omega)}\left\|h z_{\alpha}\right\|_{H^{2}(\Omega)} .
$$

Using the facts that [16]

$$
\left|\nabla z_{\alpha}\right| \leq \frac{\left|\left[z_{h}\right]\right|}{h_{K}}
$$


and

$$
\left|D^{2} z_{\alpha}\right| \leq \frac{\left|\left[\partial_{n} z_{h}\right]\right|}{h_{K}}
$$

we can estimate $\left\|h z_{\alpha}\right\|_{H^{2}(\Omega)}$ similarly with (6.6)-(6.9) to get

$\left|M_{\alpha}\left(z_{\alpha}\right)-M_{\alpha}\left(z_{h}\right)\right| \leq C_{I}\left\|M_{\alpha}^{\prime}\left(z_{h}\right)\right\|_{H^{1}(\Omega)}\left(\left\|h z_{h}\right\|_{L_{2}(\Omega)}+\left\|\left[z_{h}\right]\right\|_{L_{2}(\Omega)}+C \max _{S \subset \partial K} h_{K}^{-1}\left\|\left[\partial_{S} z_{h}\right]\right\|_{L_{2}(\Omega)}\right) \forall z_{h} \in W_{h}$.

In the case when $z_{h} \in V_{h}$ all terms with jumps in (7.9) disappear and we get estimate (7.2).

8. Mesh refinement recommendations. Using Theorems 1 and 2 we now formulate our mesh refinement recommendations which are used in Section 10 to improve accuracy of the reconstruction of the regularized solution $z_{\alpha}$ of the problem $\mathbf{P}$.

The estimate (6.1) of the Theorem 1 provides an idea on where we need to refine the mesh locally in order to improve the accuracy of the regularized solution $z_{\alpha}$. Given a finite element approximation $z_{h} \in W_{h}$ of the regularized solution $z_{\alpha} \in H^{2}(\Omega)$ the main impact of the norms of the computed finite element solution $z_{h}$ in the estimate (6.1) are provided by neighborhoods of thus points in the finite element mesh $T$ where $\left\|h z_{h}\right\|_{L_{2}(\Omega)}$ achieves its maximal value. We note that additional terms with jumps in the estimate (6.1) disappear in the case of the conforming finite element meshes when $z_{h} \in V_{h}$. Thus, the first idea of the finite element mesh refinement is that the neighborhoods of all points in the finite element mesh $T$ where the function $\left|h z_{h}\right|$ achieves its maximum should be refined.

Similarly, the estimate (7.1) of the Theorem 2 provides the second idea where to refine the finite element mesh locally in order to improve the accuracy in the Tikhonov functional (2.8). Given a finite element approximation $z_{h} \in W_{h}$ of the regularized solution $z_{\alpha} \in H^{2}(\Omega)$ and the computed Frechét derivative $M_{\alpha}^{\prime}\left(z_{h}\right)$ of the Tikhonov functional (2.8) the main impact of the norms given in the right hand side of the estimate (7.1) are provided by neighborhoods of thus points in the finite element mesh $T$ where both norms $\left\|h z_{h}\right\|_{L_{2}(\Omega)}$ and $\left\|M_{\alpha}^{\prime}\left(z_{h}\right)\right\|_{H^{1}(\Omega)}$ achieves its maximal values. Thus, the second idea of the finite element mesh refinement is that the neighborhoods of all points in the finite element mesh $T$ where $\left|M_{\alpha}^{\prime}\left(z_{h}\right)\right|$ achieves its maximum, or both functions $\left|h z_{h}\right|$ and $\left|M_{\alpha}^{\prime}\left(z_{h}\right)\right|$ achieves their maximum, should be refined.

Since the term $\left|h z_{h}\right|$ is already included in the first mesh refinement recommendation, we write only one term $\left|M_{\alpha}^{\prime}\left(z_{h}\right)\right|$ in the second mesh refinement recommendation. Thus, by combining the first and second mesh refinement recommendations we can perform numerical tests to check performance of different mesh refinement criteria.

Since Theorems 1 and 2 are valid for the common case of the functional $J_{\alpha}(z)$ defined in (2.13), we write the second mesh refinement recommendation for this functional.

The First Mesh Refinement Recommendation. Using the Theorem 1 we conclude that we should refine the mesh in neighborhoods of those points in $\Omega$ where the function $\left|h z_{h}\right|$ attains its maximal values. More, precisely in such subdomains of $\Omega$ where

$$
\left|h z_{h}\right| \geq \tilde{\varkappa} \max _{\Omega}\left|h z_{h}\right|
$$

where $\tilde{\varkappa} \in(0,1)$ is the number which should be chosen computationally and $h$ is the mesh function of the finite element mesh $T$.

The Second Mesh Refinement Recommendation. Using the Theorem 2 we conclude that we should refine the mesh in neighborhoods of those points in $\Omega$ where the function $\left|J_{\alpha}^{\prime}\left(z_{h}\right)\right|$ attains its maximal values. More precisely, let $\varkappa \in(0,1)$ be the tolerance number which should be chosen in computational experiments. Refine the mesh in such subdomains of $\Omega$ where

$$
\left|J_{\alpha}^{\prime}\left(z_{h}\right)\right| \geq \varkappa \max _{\Omega}\left|J_{\alpha}^{\prime}\left(z_{h}\right)\right| .
$$


9. The Adaptive Algorithm. In this section we present two adaptive algorithms for the solution of a Fredholm integral equation of the first kind (2.4) which we apply in numerical examples of Section 10. In our algorithms we define the minimizer and its computed solution, or approximation, on $k$ times refined mesh $T_{k}$ by $z_{\alpha}$ and $z_{k}$, correspondingly. In our mesh refinement recommendations we need compute the function $z_{k}$. To do that we use the convolution theorem (10.9) of Section 10. In Algorithm 1 we apply modified second mesh refinement recommendation of Section 8, while in Algorithm 2 we use both mesh refinement recommendations of Section 8. These algorithms are successfully tested in numerical examples of Section 10.

\section{Algorithm 1}

Step 0. Choose an initial mesh $T_{0}$ in $\Omega$ and obtain the numerical solution $z_{0}$ of (2.8) on $T_{0}$ using the finite element discretization of (4.1) for CG(1) or (4.2) for DG(1) and discretization of convolution theorem (10.9). Compute the sequence $z_{k}, k>0$, on a refined meshes $T_{k}$ via following steps:

Step 1. Obtain the numerical solution $z_{k}$ of (2.8) on $T_{k}$ using the finite element discretization of (4.1) for CG(1) or (4.2) for DG(1) and discretization of (10.9).

Step 2. Refine the mesh $T_{k}$ at all points where

$$
\left|B_{h}\left(z_{k}\right)\right| \geq \beta_{k} \max _{\Omega}\left|B_{h}\left(z_{k}\right)\right|
$$

where a posteriori error indicator $B_{h}\left(z_{k}\right)$ we define as

$$
B_{h}\left(z_{k}\right)=\int_{\Omega} z_{k}(y)\left(\int_{\Omega} \rho(x, y) \rho(x, s) d x\right) d y-\int_{\Omega} \rho(x, s) u(x) d x .
$$

Here the tolerance number $\beta_{k} \in(0,1)$ is chosen by the user.

Step 3. Construct a new mesh $T_{k+1}$ in $\Omega$ and perform steps 1-3 on the new mesh. Stop mesh refinements when $\left\|z_{k}-z_{k-1}\right\|<\epsilon$ or $\left\|B_{h}\left(z_{k}\right)\right\|<\epsilon$, where $\epsilon$ is tolerance chosen by the user. To compute norms $\left\|z_{k}-z_{k-1}\right\|$, the solution $z_{k-1}$ is interpolated from the mesh $T_{k-1}$ to the mesh $T_{k}$.

\section{Algorithm 2}

Step 0. Choose an initial mesh $T_{0}$ in $\Omega$ and obtain the numerical solution $z_{0}$ of (2.8) on $T_{0}$ using the finite element discretization of (4.1) for CG(1) or (4.2) for DG(1) and of the convolution theorem (10.9). Compute the sequence $z_{k}, k>0$, via following steps:

Step 1. Obtain the numerical solution $z_{k}$ of (2.8) on $T_{k}$ using the finite element discretization of (4.1) for CG(1) or (4.2) for DG(1) and discretization of (10.9).

Step 2. Refine the mesh $T_{k}$ at all points where

$$
\left|B_{h}\left(z_{k}\right)\right| \geq \beta_{k} \max _{\Omega}\left|B_{h}\left(z_{k}\right)\right|
$$

with a posteriori error indicator $B_{h}\left(z_{k}\right)$ defined by (9.2), and where

$$
\left|z_{k}(x)\right| \geq \widetilde{\varkappa_{k}} \max _{\Omega}\left|z_{k}(x)\right| .
$$

Here the tolerance numbers $\beta_{k}, \widetilde{\varkappa_{k}} \in(0,1)$ are chosen by the user.

Step 3. Construct a new mesh $T_{k+1}$ in $\Omega$ and perform steps 1-3 on the new mesh. Stop mesh refinements when $\left\|z_{k}-z_{k-1}\right\|<\epsilon$ or $\left\|B_{h}\left(z_{k}\right)\right\|<\epsilon$, where $\epsilon$ is tolerance chosen by the user. To compute norms $\left\|z_{k}-z_{k-1}\right\|$, the solution $z_{k-1}$ is interpolated from the mesh $T_{k-1}$ to the mesh $T_{k}$.

\section{Remarks}


- 1. We note that the choice of the tolerance numbers $\beta_{k}, \widetilde{\varkappa_{k}}$ in (9.2), (9.4) depends on the concrete values of $\max _{\Omega}\left|B_{h}\left(z_{k}\right)\right|$ and $\max _{\Omega}\left|z_{k}(x)\right|$, correspondingly. If we would choose $\beta_{k}, \widetilde{\varkappa_{k}}$ very close to 1 then we would refine the mesh in very narrow region of the computational domain $\Omega$, and if we will choose $\beta_{k}, \widetilde{\varkappa_{k}} \approx 0$ then almost all mesh of the domain $\Omega$ will be refined what is unsatisfactory. Thus, the values of the numbers $\beta_{k}, \widetilde{\varkappa_{k}}$ should be chosen in optimal way. Our numerical tests of Section 10 show that the choice of $\beta_{k}, \widetilde{\varkappa_{k}}=0.5$ is almost optimal one since with those choice of the parameters $\beta_{k}, \widetilde{\varkappa_{k}}$ the finite element mesh is refined exactly at the places where we computed the function $z_{h}$, see Figures 10.1, 10.2, 10.4. However, such choice of numbers $\beta_{k}$, $\widetilde{\varkappa_{k}}$ is valid for our computational tests of Section 10 and it can be changed during the iterations in adaptive algorithms from coarser to refined mesh.

- 2. We also note that we neglect the computation of the regularization term in a posteriori error indicator (9.2) since this term is very small and does not affects on the refinement procedure. However, this term is included in the minimization procedure of the Tikhonov's functional (2.8).

10. Numerical studies of the adaptivity technique in microtomography. In all our tests of this section we consider the problem of the two-dimensional reconstruction of the backscattered signal in microtomography $[19,20]$. This method allows nondestructive layer-by-layer image restoration of micro and nanostructures, for example, reconstruction of integral microschemes.

In the numerical tests of this section we show examples of the image restoration in microtomography using adaptive finite element method with piecewise linear functions on a locally refined meshes. We present numerical studies of the adaptive algorithms of Section 9 on the computationally simulated data (Tests 1,2 in subsection 10.2) and on the experimental data (Test 3 in subsection 10.2). In Test 2 of subsection 10.2 we also compare results obtained by the uniform deconvolution algorithm of $[19,20]$ with the adaptivity technique of this paper.

Experimental backscattered signal in Test 3 of subsection 10.2 is generated by the microtomograph developed by professor Eduard Rau at Moscow Lomonosov State University. This device is based on the electron microscope working in the backscattering electron mode. As soon as original measured images are obtained by the microtomograph they can be improved by mathematical methods. It is well known, that in mathematics the problem of the image restoration in microtomography consists in solution of a Fredholm integral equation of the first kind which is ill-posed problem. Usual method for solution of this equation in two and three dimensions is minimization of the Tikhonov functional (2.8) in some bounded domain $\Omega \subset \mathbb{R}^{n}, n=2,3$ using deconvolution algorithm on the uniform mesh $[19,20]$.

In Test 4 of subsection 10.4 we compare three different reconstruction methods: an adaptive finite element method of this paper, uniform grid deconvolution method of [19], and Bounded Total Variation method of [20]. Our tests show that the relative computational error in an adaptive finite element method is smaller than in other two methods in the case of the reconstruction of high-intensive smeared images. However, the Bounded Total Variation Method of [20] gives the smallest relative error in the case of increasing of the noise level in the computational data on the reconstruction of non-intensive smeared images.

We can conclude that the choice of the numerical reconstruction method depends on the concrete real application, level of the noise in computational data and smearing intensity of the image to be reconstructed.

10.1. Statement of the problem. Reconstruction in microtomography is based on the analyzing of energy loss of the electrons backscattered by a layer of some solid object under investigation. Usually electron probe (monokinetic electron ray) falls normally to the surface of the object. Since electrons are reflected from different depths of the object they have different energies such that we can filter them using these energies. Thus, we can get the backscattered signal only from the depth in which we are interested.

The measured intensity of the signal depends on the scattering coefficient of the material of the object. This intensity is saved into the point of a plane image which corresponds to the position of the electron probe. Collecting all intensities at all points over the domain where we measure our signal into a some matrix we can obtain the image of the distribution of the intensity in the domain under investigation.

Unfortunately, the image formed in this way has drawbacks. One of them is the blurring which can be explained by the fact that the finite radius of the electron probe leads to the situation, when every position 
of the electron probe produces some spot in the matrix, where the signal is saved.

Let us denote by $r_{0}$ the the known radius of the primary electron beam outside the target. The radius $r$ of the electron probe on the penetration depth $d$ can be represented using the empiric formula

$$
r^{\prime}(d)^{2}=r_{0}^{2}+0.625\left(\frac{Z}{E_{0}}\right)\left(\frac{k}{A}\right)^{0.5} d^{1.5},
$$

where $Z$ and $A$ are the atomic number and the atomic weight, respectively. Here, $r^{\prime}$ and $d$ are expressed in centimeters $[\mathrm{cm}]$, energy $E_{0}$ - in kelvin $[\mathrm{keV}]$, the density of the material of the target $k$ - in gram $/$ centimeter $^{3}$ $\left[\mathrm{g} / \mathrm{cm}^{3}\right]$.

The distribution of the current density in the cross section of the electron probe can be represented by a Gauss distribution [20]. This distribution transforms on the depth $d$ with the scaling parameter (dispersion) $r^{\prime}(d)$ defined by (10.1). For short notations we will define by $r(d)$ the radius of the electron probe. Thus, the distribution of the current density $\rho(r)$ can be calculated using the expression

$$
\rho(r)=\frac{1}{2 \pi r^{\prime 2}} \exp \left(-\frac{r^{2}}{2{r^{\prime}}^{2}}\right) .
$$

The distribution of the current density $\rho\left(x_{1}, x_{2}\right)$ in the Cartesian coordinate system $\left(x_{1} O x_{2}\right)$ which is defined in the plane of the layer under investigation, can be defined by the formula

$$
\rho\left(x_{1}, x_{2}\right)=\frac{1}{2 \pi r^{\prime 2}} \exp \left(-\frac{x_{1}^{2}+x_{2}^{2}}{2{r^{\prime}}^{2}}\right) .
$$

The signal distribution via the frame is formed line by line through the integral measuring of the intensity of backscattered electron signal. The measured intensity saved into the point of a plane image, which corresponds to the position of the electron probe.

Let $z\left(x_{1}, x_{2}\right)$ be the scattering coefficient at the point with coordinates $\left(x_{1}, x_{2}\right)$. Let $(\xi O \eta)$ be other Cartesian coordinate system which is defined in the plane of the image. Without loss of generality we can assume, that this system is equivalent to the system $\left(x_{1} O x_{2}\right)$. If the electron probe is located at some point $(\xi, \eta)$ of the layer under investigation, then the intensity at this point is proportional to the number of electrons backscattered from the object.

Let $\Omega$ will be the bounded domain representing the object to be investigated. We decompose this domain into $N$ small rectangular subdomains $\omega_{i}$ such that $\Omega=\cup_{i=1}^{N} \omega_{i}, \quad \omega_{i} \cap \omega_{j}=0 \quad \forall i \neq j$. Let subdomains $\omega_{i}$ have sizes $d x_{1}, d x_{2}$ such that $\omega_{i}=d x_{1} \times d x_{2}$ and the point $\left(x_{1}, x_{2}\right) \in \omega_{i}$. We assume a constant scattering coefficient $z\left(x_{1}, x_{2}\right)$ inside every subdomain $\omega_{i}$ when $d x_{1} \rightarrow 0$ and $d x_{2} \rightarrow 0$. Then the backscattered signal over the domain $\omega_{i}$ at the point $(\xi, \eta)$ is proportional to the current density $\rho\left(x_{1}-\xi, x_{2}-\eta\right)$ of the electron probe and to the scattering coefficient $z\left(x_{1}, x_{2}\right)$ at the point $\left(x_{1}, x_{2}\right)$. This signal can be described by equation

$$
u(\xi, \eta)=z\left(x_{1}, x_{2}\right) \rho\left(x_{1}-\xi, x_{2}-\eta\right) d x_{1} d x_{2} .
$$

Integrating (10.4) over all subdomains $\omega_{i} \subset \Omega$ and summing up them over the computational domain $\Omega$ we get expression for the backscattered signal $u(\xi, \eta)$ at the point $(\xi, \eta)$ in the whole domain $\Omega$

$$
u(\xi, \eta)=\int_{\Omega} z\left(x_{1}, x_{2}\right) \rho\left(x_{1}-\xi, x_{2}-\eta\right) d x_{1} d x_{2} .
$$

Inverse problem (IP)

Let the function $z\left(x_{1}, x_{2}\right) \in H^{1}$ in the equation

$$
\int_{\Omega} \rho\left(x_{1}-\xi, x_{2}-\eta\right) z\left(x_{1}, x_{2}\right) d x_{1} d x_{2}=u(\xi, \eta), \quad \xi, \eta \in \Omega,
$$

be unknown in the domain $\Omega$. Determine the function $z\left(x_{1}, x_{2}\right) \in H^{1}$ for all points $\left(x_{1}, x_{2}\right) \in \Omega$ assuming that functions $\rho\left(x_{1}-\xi, x_{2}-\eta\right) \in C^{k}(\Omega \times \Omega), k \geq 0$ and $u(\xi, \eta) \in L_{2}(\Omega)$ in (10.6) are known. 
We note that the equation (10.6) is a Fredholm integral equation of the first kind in two dimensions. It can be presented in the form (2.2) with the convolution operator $A: H^{1} \rightarrow L_{2}(\Omega)$ defined by

$$
A(z):=\int_{\Omega} \rho\left(x_{1}-\xi, x_{2}-\eta\right) z\left(x_{1}, x_{2}\right) d x_{1} d x_{2} .
$$

To solve the inverse problem IP we minimize the Tikhonov functional (2.8) in the form

$$
M_{\alpha}(z)=\left\|\int_{\Omega} \rho\left(x_{1}-\xi, x_{2}-\eta\right) z\left(x_{1}, x_{2}\right) d x_{1} d x_{2}-u(\xi, \eta)\right\|_{L_{2}(\Omega)}^{2}+\alpha\left\|z\left(x_{1}, x_{2}\right)\right\|_{H^{1}}^{2} .
$$

Using the convolution theorem and properties of a Fourier transform [25, 26] similarly with (2.10) we can obtain the following formula of the minimizer $z_{\alpha}\left(x_{1}, x_{2}\right)$ of the functional (10.8) in two dimensions

$$
z_{\alpha}\left(x_{1}, x_{2}\right)=\int_{\mathbb{R}^{2}} e^{-i\left(\lambda x_{1}+\nu x_{2}\right)} \frac{\hat{\rho}^{*}(\lambda, \nu) \hat{u}(\lambda, \nu)}{|\hat{\rho}(\lambda, \nu)|^{2}+\alpha\left(1+\lambda^{2}+\nu^{2}\right)} d \lambda d \nu,
$$

where functions $\hat{u}$ and $\hat{\rho}$ are the Fourier transforms of the functions $u$ and $\rho$, respectively.

In $[20,21]$ the solution of the equation (10.5) was found on uniform grids on the spaces $H^{1}$ and $V H(B)$ (the space of functions with bounded total variation). The best reconstruction result was obtained using the bounded total variation functions when for the first approximation was taken the result obtained on space $H^{1}$. Our computational tests of Section 10.2 show the better stability for the adaptive method than methods of $[20,21]$.

10.2. Tests with simulated backscattered data. The goal in Test 1 and Test 2 was to solve the problem IP and restore the function $z\left(x_{1}, x_{2}\right)$ in (10.6) under the condition that we know functions $\rho\left(x_{1}-\right.$ $\left.\xi, x_{2}-\eta\right) \in C^{k}(\Omega \times \Omega), k \geq 0$ and $u(\xi, \eta) \in L_{2}(\Omega)$ in (10.6). The function $\rho\left(x_{1}-\xi, x_{2}-\eta\right)$ is given by (10.3), and the function $u(\xi, \eta)$ represents the backscattered signal. In all our tests we decompose the finite element domain $\Omega$ into non-overlapping triangles as described in Section 3.

In Tests 1-3 we used simulated backscattered data $u(\xi, \eta)$. More precisely, we compute the function $u(\xi, \eta)$ in (10.6) using the known functions $z\left(x_{1}, x_{2}\right)$ and $\rho\left(x_{1}-\xi, x_{2}-\eta\right)$ in (10.6). Next, the function $z\left(x_{1}, x_{2}\right)$ is "forgotten" and our goal is to reconstruct this function for all $\left(x_{1}, x_{2}\right) \in \Omega$ by known functions $\rho\left(x_{1}-\xi, x_{2}-\eta\right)$ and $u(\xi, \eta) \in L_{2}(\Omega)$ in $(10.6)$.

We define a posterori error indicator (9.2) for the problem IP as

$$
\begin{aligned}
B_{h}\left(z_{k}\right) & =\int_{\Omega} z_{k}(\xi, \eta)\left(\int_{\Omega} \rho\left(x_{1}-\xi, x_{2}-\eta\right) \rho\left(x_{1}-s_{1}, x_{2}-s_{2}\right) d x_{1} d x_{2}\right) d \xi d \eta \\
& -\int_{\Omega} \rho\left(x_{1}-s_{1}, x_{2}-s_{2}\right) u\left(x_{1}, x_{2}\right) d x_{1} d x_{2},
\end{aligned}
$$

where $\Omega$ is our two-dimensional computational domain. Recall that according to the Remark 2 we omit computation of the regularization term in the indicator (10.10) since this term is negligible compared to other terms presented in this indicator. Thus, this term does not affect into refinement of the mesh.

10.2.1. Test 1-a). The computational domain $\Omega$ in this test has the area $S_{\Omega}=3.4813 \mathrm{mkm}(1 \mathrm{mi}$ crometre $\mathrm{mkm}=1 \cdot 10^{-6}$ of a metre). The backscattered signal is the simulated function $u(\xi, \eta)$ in $(10.6)$ which is presented in Figure 10.1-a). This function is computed for the function $\rho\left(x_{1}-\xi, x_{2}-\eta\right)$ with the smearing parameter $r^{\prime}=0.188 \mathrm{mkm}$ in (10.2).

To reconstruct the function $z\left(x_{1}, x_{2}\right)$ for backscattered data given in Figure 10.1-a) we apply the first adaptive algorithm of Section 9. First, we compute the function $z_{0}$ on a coarse mesh $T_{0}$ for $k=0$ with the regularization parameter $\alpha=3 e 10-07$ in (10.8). The coarse mesh $T_{0}$ is presented in Figure 10.1-g). 


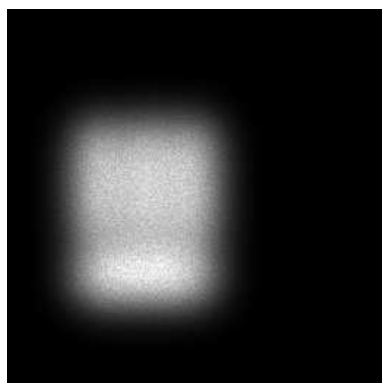

a) 7938 elements

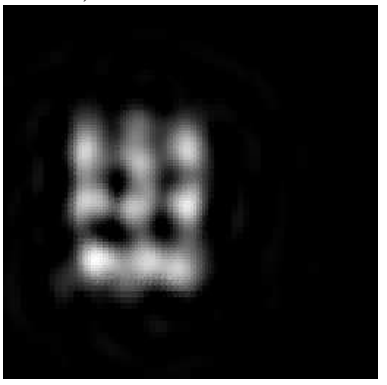

d) $z_{3}, 17706$ elements

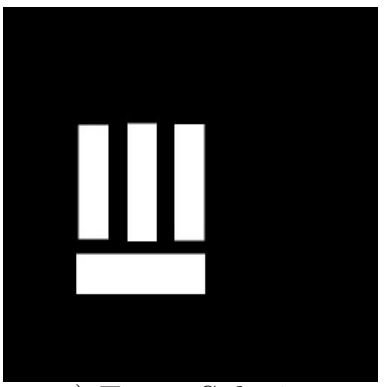

g) Exact Solution

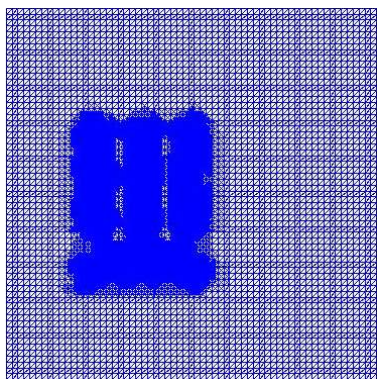

j) 17706 elements

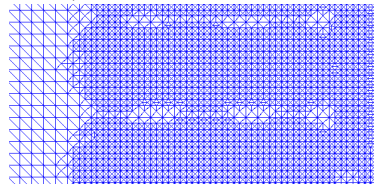

m)

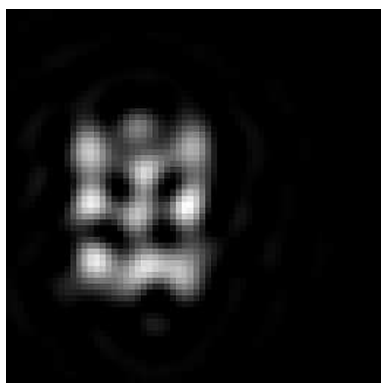

b) $z_{1}, 9336$ elements

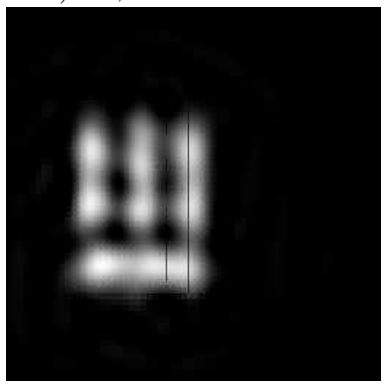

e) $z_{4}, 28676$ elements

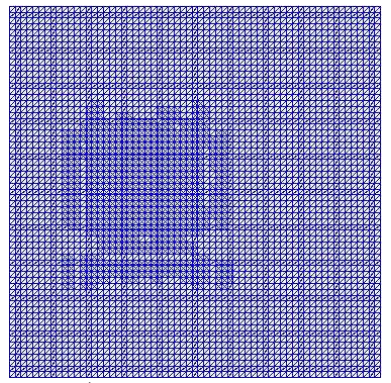

h) 9336 elements

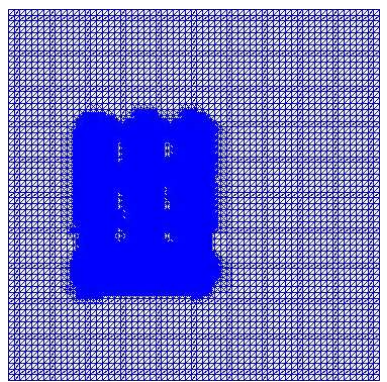

k) 28676 elements

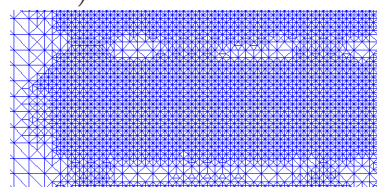

n)

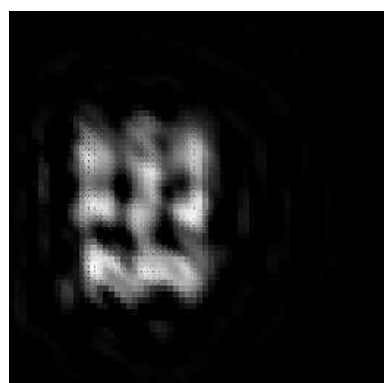

c) $z_{2}, 12094$ elements

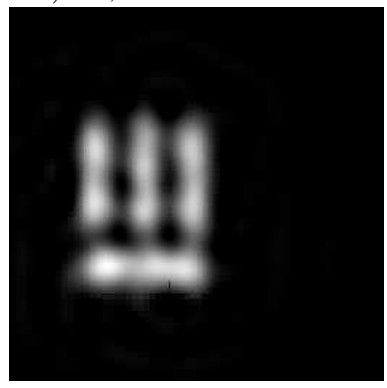

f) $z_{5}, 48864$ elements

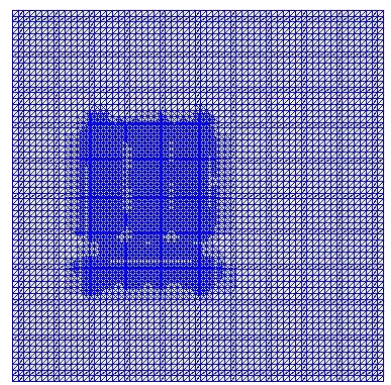

i) 12094 elements

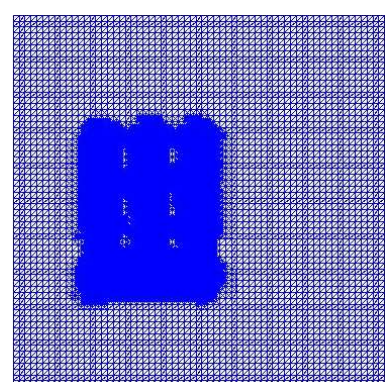

1) 48864 elements

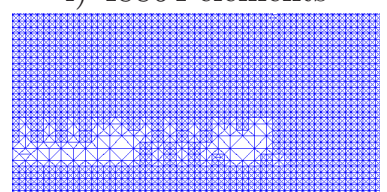

o)

Fig. 10.1. Test 1-a). Reconstruction of the function $z\left(x_{1}, x_{2}\right)$ using simulated backscattered data. On a) we present simulated backscattered data $u(\xi, \eta)$. On $b)-f)$ we show results of the reconstruction of the function $z\left(x_{1}, x_{2}\right)$ on different adaptively refined meshes using the algorithm of Section 9, on g) we present the known exact function $z^{*}$. Adaptively refined meshes corresponding to the images on c)-f) are presented on $h$ )-l). Enlarged parts of the refined meshes on $j), k$ ), l) are presented on $m), n$ ), o), respectivel $y$. 


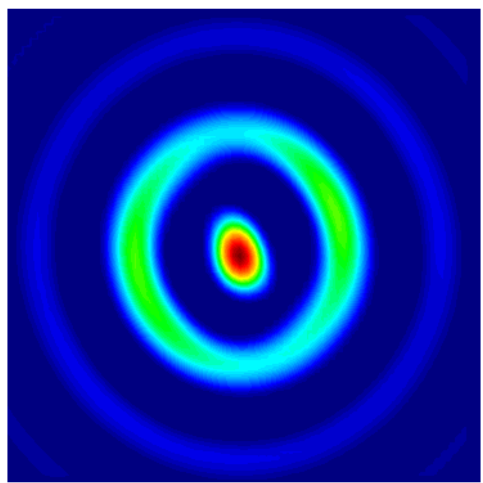

a) 7938 elements

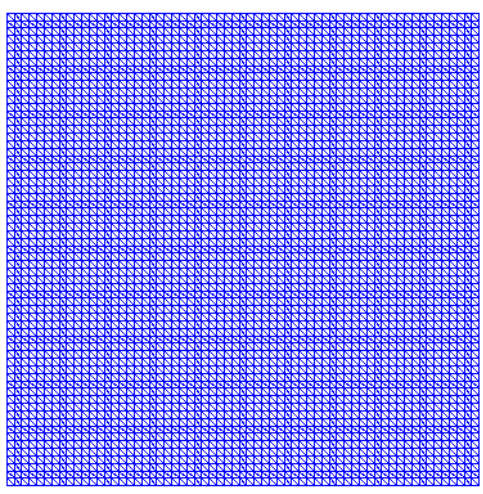

d) 7938 elements

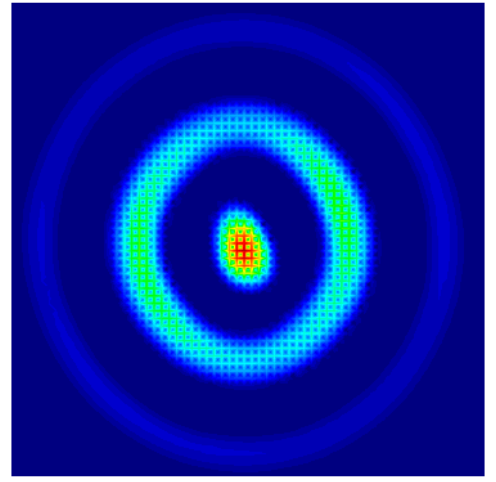

b) $z_{1}, 9604$ elements

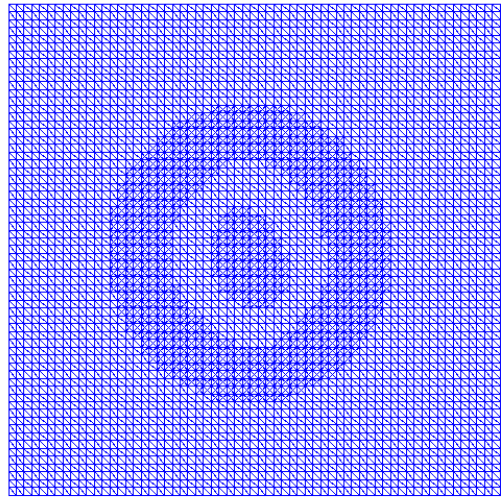

e) 9604 elements

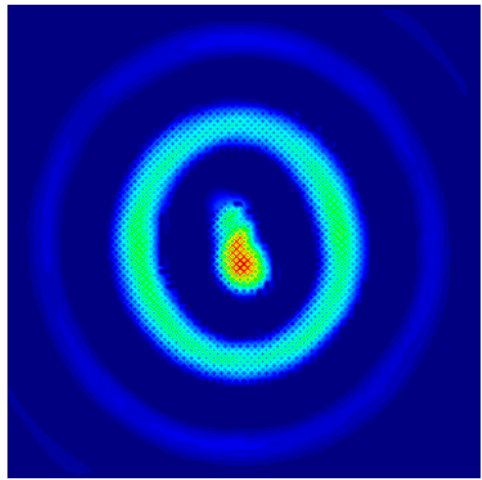

c) $z_{2}, 12798$ elements

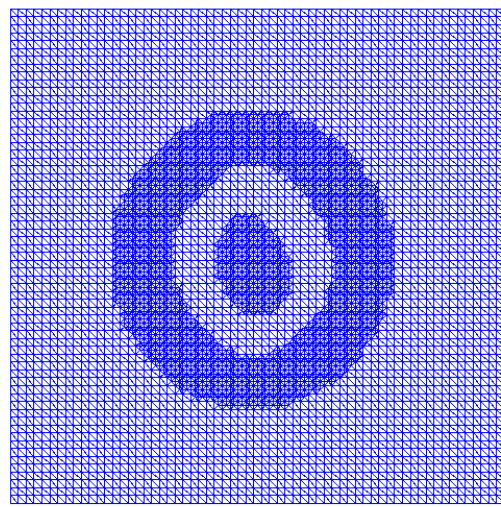

f) 12798 elements

Fig. 10.2. Test 1-b). Reconstruction of the function $z\left(x_{1}, x_{2}\right)$ using simulated backscattered data. On a) we present simulated backscattered data $u(\xi, \eta)$. On $b)-f)$ we show results of the reconstruction of the function $z\left(x_{1}, x_{2}\right)$ on the different adaptively refined meshes using the second algorithm of Section 9. Adaptively refined meshes corresponding to the images on $b$ )-c) are presented on e)-f).

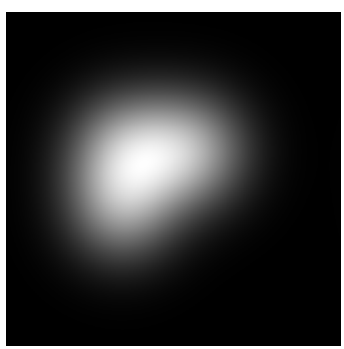

a)

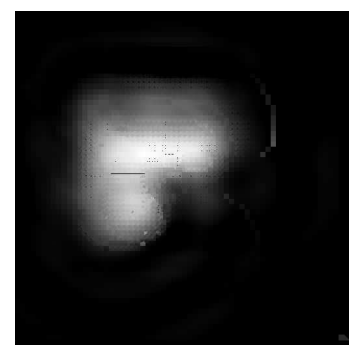

b)

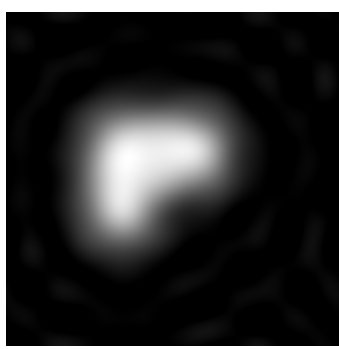

c)

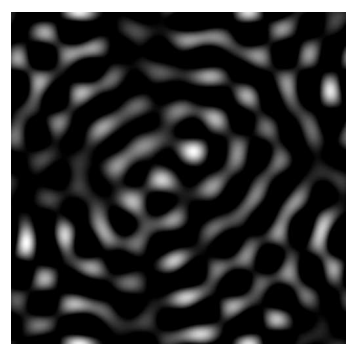

d)

Fig. 10.3. Test 2. Efficiency of the application of the adaptive mesh refinement for calculation of the deconvolution function $z_{\alpha}$ given by (10.9). a) Simulated backscattered data $u(\xi, \eta)$. b) Computed deconvolution function $z_{\alpha}$ on the adaptively refined mesh with the regularization parameter $\alpha=2 e 10-7$. c),d) Computed deconvolution function $z_{\alpha}$ on the uniform mesh with the regularization parameters $\alpha=0.01$ and $\alpha=2 e 10-7$, respectively.

Now we describe our stopping criterion for mesh refinements. According to the second mesh refinement recommendation we refine the mesh in all subdomains where the function $B_{h}\left(z_{k}\right)$ attains its maximal values, 


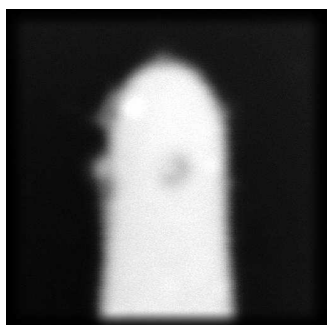

a) 7938 elements

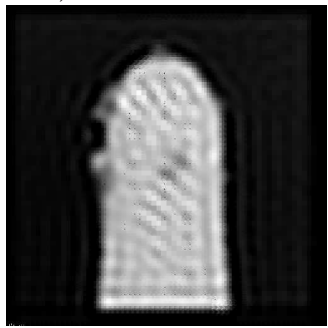

d) $z_{3}, 24262$ elements

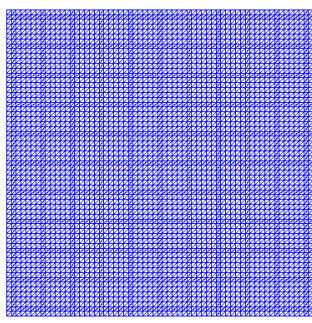

g) 7938 elements

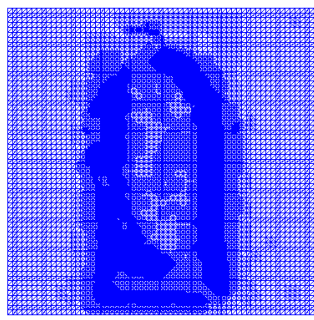

j) 24262 elements

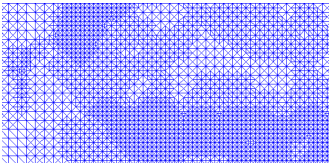

$\mathrm{m})$

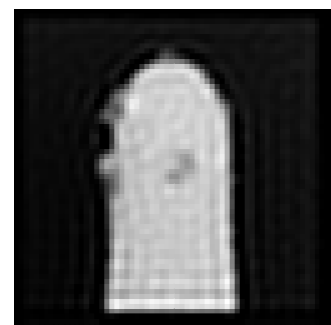

b) $z_{1}, 11270$ elements

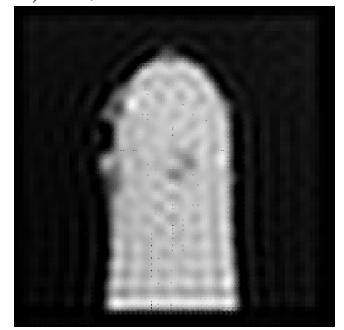

e) $z_{4}, 40358$ elements

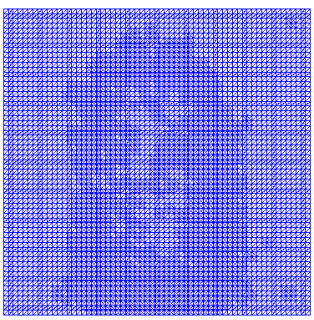

h) 11270 elements

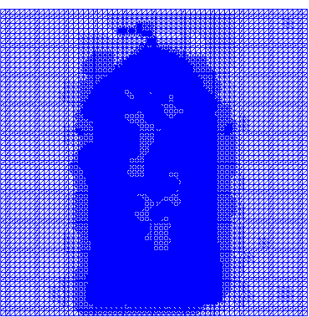

k) 40358 elements

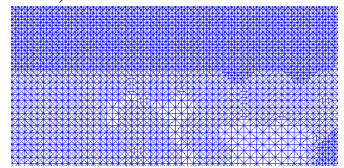

n)

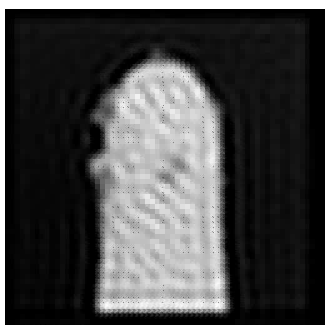

c) $z_{2}, 15916$ elements

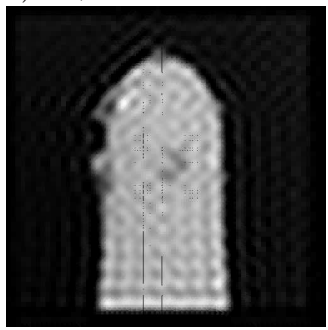

f) $z_{5}, 72292$ elements

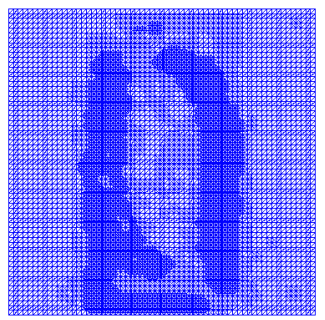

i) 15916 elements

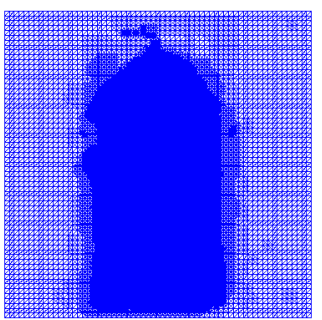

1) 72292 elements

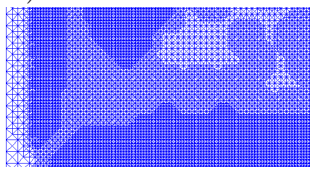

o)

FIG. 10.4. Test 3. Reconstruction of the function $z\left(x_{1}, x_{2}\right)$ from the experimental backscattering data obtained by the microtomograph [19, 20]. On a) we present the real measured signal $u(\xi, \eta)$ on the part of the planar microscheme $\Omega$ obtained by microtomograph on the depth $0.9 \mu \mathrm{m}$. On b)-f) we show results of the reconstruction of the function $z\left(x_{1}, x_{2}\right)$ on different adaptively refined meshes using the algorithm of Section 9. Adaptively refined meshes corresponding to the images on $b)-f$ ) are presented on $h$ )-l). Enlarged parts of refined meshes on $j$ ), $k$ ), l) are presented on $m), n), o)$, respectively. 
or where

$$
\left|B_{h}\left(z_{k}\right)\right| \geq \beta_{k} \max _{\Omega}\left|B_{h}\left(z_{k}\right)\right|
$$

with $\beta_{k}=0.5$. Next, we perform all steps of the first adaptive algorithm of Section 9 until the desired tolerance $\left\|z_{k}-z_{k-1}\right\|<\epsilon$ with $\epsilon=10 e-05$ is achieved, or the computed $L_{2^{-}}$norms of the differences $\left\|z_{k}-z_{k-1}\right\|$ start to grow abruptly.

Figures 10.1-b)-f) show results of the reconstruction of the function $z\left(x_{1}, x_{2}\right)$ on the adaptively refined meshes presented in Figures 10.1-h)-l). We observe that on the fifth refined mesh corresponding to the Figure 10.1-l) we obtain the best restoration results. Since the computed $L_{2^{-}}$norms $\left\|z_{k}-z_{k-1}\right\|$ with $k=5$ start to grow abruptly after the fifth refinement of the initial mesh we conclude that the function $z_{5}$ of the Figure 10.1-f) is our final reconstruction result.

10.2.2. Test 1-b). This test is similar to the previous one only the goal was to reconstruct the function $z\left(x_{1}, x_{2}\right)$ in (10.6) using backscattered data $u(\xi, \eta)$ presented in Figure 10.2-a). The computational domain $\Omega$ in this test is the same as in Test 1-a). The backscattered signal of Figure 10.2-a) is the simulated function $u(\xi, \eta)$ with the same smearing parameter $r^{\prime}=0.188 \mathrm{mkm}$ in (10.2) as in Test 1-a).

To reconstruct the function $z\left(x_{1}, x_{2}\right)$ we apply the second adaptive algorithm of Section 9 with both mesh refinement recommendations of Section 8. Figures 10.2-b)-c) show results of the reconstruction of the function $z\left(x_{1}, x_{2}\right)$ on the adaptively refined meshes presented in Figures 10.2-e)-f). Stopping criterion for mesh refinements is the same as in the Test 1-a).

We observe that on the second refined mesh corresponding to Figure 10.2-f) we obtain the best restoration results. Since the computed $L_{2^{-}}$norms $\left\|z_{k}-z_{k-1}\right\|$ start to grow abruptly after the second refinement of the initial mesh we conclude that the function $z_{2}$ of Figure 10.2-c) is our final reconstruction result.

10.2.3. Test 2. The goal of this test is to present the efficiency and robustness of applying of adaptive mesh refinement for calculating of deconvolution function $z_{\alpha}$ given by (10.9). The computational domain $\Omega$ in this test has the area $S_{\Omega}=6.963 \mathrm{mkm}$. The backscattered signal is the simulated function $u(\xi, \eta)$ in (10.6) which is presented in Figure 10.3-a). This function is computed for the function $\rho\left(x_{1}-\xi, x_{2}-\eta\right)$ with the smearing parameter $r^{\prime}=0.0612 \mathrm{mkm}$ in (10.2). On Figure 10.3-b) we present computed deconvolution function $z_{\alpha}$ on the adaptively refined mesh using the first adaptive algorithm of Section 9 with the small regularization parameter $\alpha=2 e 10-7$ in the Tikhonov functional (10.8). Figure 10.3-c) shows computed deconvolution function $z_{\alpha}$ on the uniform mesh with the regularization parameter $\alpha=0.01$ in the Tikhonov functional (10.8). Figure 10.3-d) shows that the image blows up on the uniform mesh taking the small regularization parameter $\alpha=2 e 10-7$ in the Tikhonov functional (10.8).

Comparing results of Figures 10.3 we can conclude that the computed deconvolution function $z_{\alpha}$ on the adaptively refined mesh of the Figure 10.3-b) is better than the corresponding function on the uniform mesh of the Figure 10.3-c). We observe, that this function blows up on the uniform mesh when the regularization parameter is taken the same as in the adaptive algorithm, compare results of Figure 10.3-b) with the Figure 10.3-d). This test points out towards the robustness of using the adaptive algorithm for image restoration problems.

10.3. Tests with experimental data. Test 3. In this test our goal was to solve the problem IP and restore the function $z\left(x_{1}, x_{2}\right)$ in (10.6) using experimentally measured backscattered data $u(\xi, \eta)$ and the known function $\rho\left(x_{1}-\xi, x_{2}-\eta\right)$ in (10.6). The computational domain $\Omega$ in this test has the area $S_{\Omega}=16.963$ mkm and represents the part of the planar microscheme. In Figure 10.4-a) we show the backscattered signal $u(\xi, \eta)$ obtained from the experimentally measured data by microtomograph $[19,20]$. This function was measured on the depth $d=0.9 \mu \mathrm{m}$ of the microscheme with the smearing parameter $r^{\prime}=0.149 \mathrm{mkm}$ in (10.2).

To reconstruct the function $z\left(x_{1}, x_{2}\right)$ from backscattered data given in Figure 10.4-a) we apply the first adaptive algorithm of Section 9. First, we compute $z_{0}$ using the finite element discretization of (10.9) with the regularization parameter $\alpha=2 e 10-07$ in (10.8) on the coarse mesh presented in Figure 10.4-g). Then we refine the mesh in all subdomains where the gradient of (10.10) attains its maximal values by 
choosing $\beta_{k}=0.5$ in (10.11). Next, we perform all steps of the adaptive algorithm until the desired tolerance $\left\|z_{k}-z_{k-1}\right\|<\epsilon$ with $\epsilon=10 e-05$ is achieved or the computed $L_{2^{-}}$norms of the differences $\left\|z_{k}-z_{k-1}\right\|$ start to grow abruptly.

Figures 10.4-b)-f) show results of the reconstruction of the function $z\left(x_{1}, x_{2}\right)$ on the adaptively refined meshes which are presented in Figures 10.4-h)-l). We observe that on the fifth refined mesh corresponding to the Figure 10.4-1) we obtain the best reconstruction results. Since the computed $L_{2}$ - norms $\left\|z_{k}-z_{k-1}\right\|$ start to grow abruptly after the fifth refinement of the initial mesh we conclude that the function $z_{5}$ of Figure 10.4-f) is our final reconstruction result.

10.4. Test 4: comparison with other methods. In this section we present performance comparison between three different reconstruction methods: adaptive finite element method of this paper, uniform grid deconvolution method on the Sobolev space $H^{1}$ of [19], and Bounded Total Variation method $V H$ of [20].

In all our tests we solve the problem IP and reconstruct the function $z\left(x_{1}, x_{2}\right)$ in (10.6) using the backscattered function $u(\xi, \eta)$ given in Figure 10.1-a) and known function $\rho\left(x_{1}-\xi, x_{2}-\eta\right)$ in (10.6). The computational domain $\Omega$ in this test is the same as in Test 1 . We perform two different set of tests: in Test 4-a we use simulated computational data with different noise level in them (we apply additive white Gaussian noise where the maximal amplitude $I_{a}$ is varied on the interval $I_{a}=[0 \% ; 100 \%]$ ), and in Test 4-b we use different values of the smearing parameter $r^{\prime}(d)$ such that this parameter belongs to the interval $r^{\prime}(d)=[0,0.3 \cdot l]$, where $l$ is the linear size of the frame under investigation.

Our analysis is based on calculating of relative error $e_{r}$

$$
e_{r}=\frac{\left\|z^{*}-z_{\alpha}\right\|_{L_{2}(\Omega)}^{2}}{\left\|z^{*}\right\|_{L_{2}(\Omega)}^{2}} .
$$

Here, $z^{*}$ defines the exact solution and $z_{\alpha}$ - computed solution of the problem IP. Note, that to compute relative error in (10.12) we used the known exact solution $z^{*}$ presented on Figure 10.1-g).

10.4.1. Test 4-a. Figure 10.5 shows performance comparison of three above named methods. Using Figure 10.5-a) we observe that the Bounded Total Variation Method gives the best performance results in Test 4-a: with increasing of the noise level this method gives smallest relative error $e_{r}$. We also observe that an adaptive finite element method performs better than the uniform grid deconvolution method with increasing of the noise level in the computational data. In this test an adaptive finite element method was applied with the piecewise-constant function in the finite element approximation of the function $z\left(x_{1}, x_{2}\right)$. We are planning to check performance of an adaptive finite element method with higher polynomial approximation of this function in the future research.

10.4.2. Test 4-b. Figure 10.5-b) shows performance results of three reconstruction methods when using different values of the smearing parameter $r^{\prime}(d)$ in computations. We observe that the smallest relative error we obtain using the adaptive finite element method when the function $r^{\prime}(d)$ has the high intensity $\left(r^{\prime}(d)>0.2\right)$. We can conclude that an adaptive FEM performs better than other two methods in the case of the reconstruction of high-intensive smeared images.

11. Conclusion. In this work we considered a problem of the solution of a Fredholm integral equation of the first kind (2.1) as an ill-posed problem $\mathbf{P}$. To find regularized solution $z_{\alpha}$ of the problem $\mathbf{P}$ we minimized the Tikhonov functional (2.8) on adaptivelly refined meshes. To do that we use CG(1) and DG(1) finite element methods to solve the minimization problem (2.14). In Theorem 1 we derived a posteriori error estimates for the error in the regularized solution $z_{\alpha}$ of the Tikhonov functional (2.8), and in Theorem 2 for the error in Tikhonov functional (2.8). Both theorems are valid for the case of more general functional (2.13). Using these theorems we formulated local mesh refinement recommendations which improve accuracy of the reconstruction of the regularized solution $z_{\alpha}$ in the Tikhonov functionals (2.8) and (2.13). Our twodimensional numerical experiments of Section 10 justify efficiency of our a posteriori estimates applied both to the computationally simulated and experimental backscattered data measured in microtomography. 


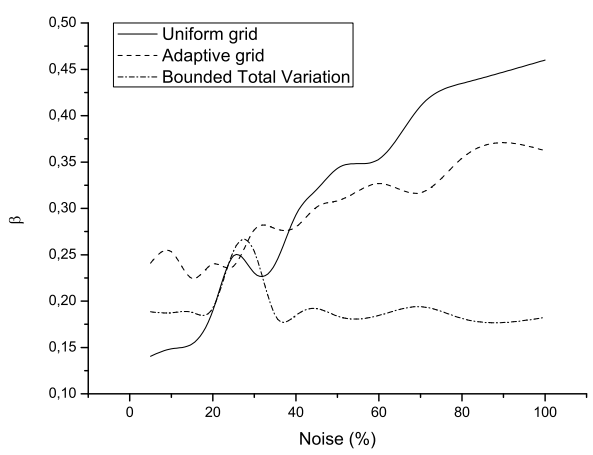

a) Test $4-\mathrm{a}$

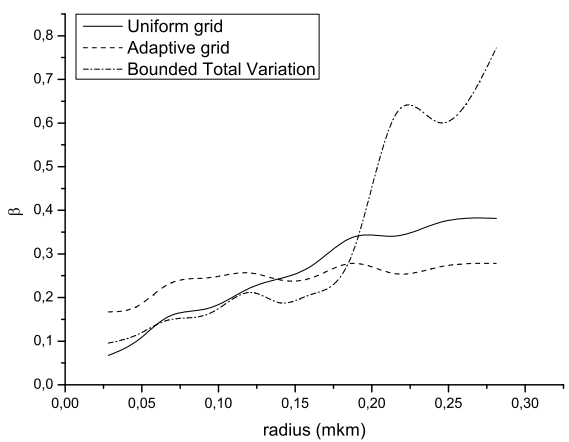

b) Test $4-b$

FIG. 10.5. Test 4. Performance comparison of an adaptive finite element method, uniform grid deconvolution method and Bounded Total Variation method.

\section{Acknowledgments}

This research was supported by the Swedish Research Council, the Swedish Foundation for Strategic Research (SSF) in Gothenburg Mathematical Modelling Centre (GMMC) and by the Swedish Institute, Visby Program. The first author acknowledges also the Russian Foundation For Basic Research, the grant RFFI 11-01-00040.

\section{REFERENCES}

[1] M. Asadzadeh, K. Eriksson, On adaptive finite element methods for Fredholm integral equations of the second kind, SIAM Journal on Numerical Analysis, V.31, N.3, pp. 831-855, 1994.

[2] K. E. Atkinson, The numerical solution of integral equations of the second kind, Cambridge University Press, 1997.

[3] Yu.A.Basistov, A.V.Goncharsky, E.E.Lekht, A.M.Cherepashchuk, A.G.Yagola. Application of the regularization method for increasing of the radiotelescope resolution power, Astron. zh., V. 56, N. 2, pp. 443-449 (in Russian), 1979.

[4] A. B. Bakushinsky, M. Y. Kokurin, A. Smirnova, Iterative methods for ill-posed problems, Walter de Gruyter GmbH\&Co., 2011.

[5] A.B. Bakushinsky, A posteriori error estimates for approximate solutions of irregular operator equations, Doklady Mathematics, 83, pp.1-2, 2011.

[6] L. Beilina, M.V. Klibanov and M.Yu. Kokurin, Adaptivity with relaxation for ill-posed problems and global convergence for a coefficient inverse problem, Journal of Mathematical Sciences, 167, pp. 279-325, 2010.

[7] L. Beilina and M.V. Klibanov, A posteriori error estimates for the adaptivity technique for the Tikhonov functional and global convergence for a coefficient inverse problem, Inverse Problems, 26, 045012, 2010.

[8] L. Beilina, M. V. Klibanov and A. Kuzhuget, New a posteriori error estimates for adaptivity technique and global convergence for a hyperbolic coefficient inverse problem, Journal of Mathematical Sciences, 172, 449-476, 2011.

[9] L.Beilina, M.V.Klibanov, Reconstruction of dielectrics from experimental data via a hybrid globally convergent/adaptive inverse algorithm, Inverse Problems, 26, 125009, 2010.

[10] A.V.Bolotina, F.A.Lukyanov, E.I. Rau, R.A. Sennov, A.G.Yagola. Energy spectra of the electrons backscattered of the massive targets, Vestnik Moskovskogo Universiteta, Section 3:Physics, Astronomy, V.5, pp.30-32, 2009.

[11] S. C. Brenner and L. R. Scott, The Mathematical Theory of Finite Element Methods (2nd edn), Springer-Verlag, New York, 2002.

[12] K. Eriksson, D. Estep and C. Johnson, Calculus in Several Dimensions, Springer, Berlin, 2004.

[13] A.V.Goncharsky, A.M.Cherepashchuk, A.G.Yagola. Ill-posed problems of astrophysics. -Moscow, Nauka, pp. 1-352 (in Russian), 1985.

[14] Gostev A.V., Ditsman S.A., Luk'janov F.A., Orlikovskij N.A., Rau E.I., Sennov R.A., Method and instrumentation microtomography in scanning electron microscopy. J. Instruments and Experimental Techniques, 4, .124-134, 2010.

[15] , Groetsch, Inverse Problems in the Mathematical Sciences, Friedr.ViewegSohn Verlagsgesellschaft, Braunschweig/Wiesbaden, Germany, 1993.

[16] C. Johnson and A. Szepessy, Adaptive finite element methods for conservation laws based on a posteriori error estimation, Comm. Pure Appl. Math., 48, 199-234, 1995.

[17] C. Johnson, Numerical Solution of Partial Differential Equations by the Finite Element Method, Dover Books on Mathematics, 2009. 
[18] M.V. Klibanov, A.B. Bakushinsky and L. Beilina, Why a minimizer of the Tikhonov functional is closer to the exact solution than the first guess, J. Inverse and Ill-Posed Problems, 19, 83-105, 2011.

[19] A.G. Yagola, N.A. Koshev. Restoration of smeared and defocused color images, Numerical Methods and Programming, V. 9, pp. 207-212 (in Russian), 2008.

[20] N.A. Koshev, N.A. Orlikovsky, E.I. Rau, A.G. Yagola. Solution of the inverse problem of restoring the signals from an electronic microscope in the backscattered electron mode on the class of bounded variation functions, Numerical Methods and Programming, V.12, pp. 362-367 (in Russian), 2011.

[21] N.A. Koshev, F.A. Luk'anov, E.I. Rau, R.A. Sennov, and A.G. Yagola. Increasing Spatial Resolution in the Backscattered Electron Mode of Scanning Electron Microscopy, Bulletin of the Russian Academy of Sciences. Physics. v. 75, No. 9, pp. 1181-1184. Allerton Press, 2011.

[22] R. Kress, Linear Integral Equations, Springer-Verlag, Berlin, 1989.

[23] V.D.Rusov, Yu.F.Babikova, A.G.Yagola. Image restoration in electronic microscopy autoradiography of surfaces. Moscow, Energoatomizdat, pp. 1-216 (in Russian), 1991.

[24] A. N. Tikhonov, A. S. Leonov, A. G. Yagola, Nonlinear ill-posed problems, Chapman \& Hall, 1998.

[25] A.N. Tikhonov, A.V. Goncharsky, V.V. Stepanov and A.G. Yagola, Numerical Methods for the Solution of Ill-Posed Problems, London: Kluwer, London, 1995.

[26] Tikhonov A.N., Goncharskiy A.V., Stepanov V.V., Kochikov I.V. Ill-posed problems of the image processing, DAN USSR, 294, 4. 832-837, 1987. 